\title{
Das schwarze Loch der „Nicht-Nachhaltigkeit““. Eine kritische Auseinandersetzung mit Ingolfur Blühdorns Forschungsansatz
}

\author{
Karl-Werner Brand
}

Angenommen: 5. Juli 2021 / Online publiziert: 3. August 2021

(C) Der/die Autor(en) 2021

Zusammenfassung Ingolfur Blühdorn hat in den vergangenen Jahren in vielen kritischen Beiträgen die These vertreten, dass wir uns entgegen den ,naiven Hoffnungserzählungen“ der transformativen Nachhaltigkeitsforschung in einem strukturell geschlossenen System der ,nachhaltigen Nicht-Nachhaltigkeit“ bewegen. Sein Forschungsinteresse zielt auf die Klärung der Frage, warum sich dieses System der „Nicht-Nachhaltigkeit“ trotz des gestiegenen Umweltbewusstseins und aller Nachhaltigkeitsaktivitäten über Jahrzehnte hinweg so stabil halten konnte und welche politischen Konsequenzen sich daraus ergeben. Der für ihn zentrale Erklärungsfaktor ist dabei - als Nebenfolge sozio-kultureller Modernisierungsdynamiken - die Radikalisierung von Selbstverwirklichungsansprüchen, die konsumtive, nicht-nachhaltige Identitäten und Lebensstile zu unverhandelbaren Voraussetzungen demokratischer Politik werden lassen. Das führe zwangsläufig zur Transformation der Demokratie in ein ,postdemokratisches“ Instrument der Verschärfung von Ungleichheit und Exklusion, was aber durch ,simulative Nachhaltigkeitspraktiken“ und „Praktiken der Selbstillusionierung“ überdeckt werde. Der Beitrag rekonstruiert die zentralen Argumentationsschritte dieses Ansatzes und diskutiert seine zentralen Annahmen, insbesondere die der „Emanzipation zweiter Ordnung“ und der ,postdemokratischen Wende“. Diese Annahmen erweisen sich, so das Ergebnis dieser Diskussion, sowohl historisch wie theoretisch als unhaltbar. Damit führt auch Blühdorns Versuch, die Widersprüchlichkeiten und Blockaden von Nachhaltigkeitstransformationen zu begreifen, ins Leere. Die Frage ist, was sich aus dem Scheitern dieses Ansatzes lernen lässt und welche Desiderate der sozialwissenschaftlichen Nachhaltigkeits- und Transformationsforschung sich daraus ergeben.

Karl-Werner Brand $(\bowtie)$

Technische Universität München, Arcisstraße 21, 80333 München, Deutschland

E-Mail: karl-werner.brand@tum.de 
Schlüsselwörter Politik der Nicht-Nachhaltigkeit · (Post-)Demokratie · Historische Subjektformen · Emanzipation zweiter Ordnung · Simulative Politik · Sozialwissenschaftliche Nachhaltigkeitsforschung · Sozial-ökologische Transformation

\title{
The black hole of unsustainability. A critical discussion of Ingolfur Blühdorn's approach
}

\begin{abstract}
For many years Ingolfur Blühdorn has advanced the thesis that we move in a structurally closed system of "sustainable non-sustainability" - quite contrary to the naive narratives of hope underlying mainstream transformation research and despite all public sustainability activities and new degrowth movements. Thus, he is not interested in the question of how barriers to sustainability could be removed best, but of how the astonishing stability of this system of "non-sustainability" can be explained. The major explanatory factor for him here - as a side effect of socio-cultural modernization dynamics - is the radicalization of self-actualization claims that makes post-modern consumerist identities and lifestyles non-negotiable prerequisites of democratic politics. This inevitably transforms democracy (as an inclusive political order that guarantees universal rights and freedoms) into a "postdemocratic" instrument of exacerbating inequality and social exclusion, which is nonetheless masked by simulative sustainability discourses and practices of selfillusion. The article reconstructs the structure of Blühdorn's argumentation and critically discusses its key elements, such as "second-order emancipation" and "postdemocratic turn". In sum, the critical discussion shows that none of these arguments are theoretically and historically convincing. Despite its "meta-critical" claim, Blühdorn's approach cannot explain the typical contradictions and barriers of sustainability transformation. The question remains what can be learned from this discussion for the understanding of the dynamics and problems of social-ecological transformations.
\end{abstract}

Keywords Politics of unsustainability - Second-order emancipation · Postdemocratic turn · Historic forms of subjectivity $\cdot$ Simulative politics $\cdot$ Sociological sustainability research $\cdot$ Socio-ecological transformation

\section{Le trou noir de la « non durabilité ». Une discussion critique de l'approche d'Ingolfur Blühdorn}

Résumé Ingolfur Blühdorn a, dans de nombreux articles critiques parus ces dernières années, défendu la thèse selon laquelle nous nous trouverions, à l'encontre des « récits naïfs porteurs d'espoir » de la recherche sur les transformations écologiques, dans un système structurellement clos de «non durabilité durable ». Ses recherches portent sur la question de savoir pourquoi ce système de «non durabilité » a pu, malgré les progrès de la conscience écologique et des activités durables, se maintenir de manière si stable pendant des décennies et quelles conséquences politiques il convient d'en tirer. Le principal facteur explicatif est selon lui la radicalisation, résultant des dynamiques de modernisation socio-culturelles, de prétentions à la réa- 
lisation de soi élevant des identités et des styles de vie consuméristes non durables au rang de conditions non négociables de la politique démocratique. La démocratie se muerait ainsi inévitablement en un instrument « postdémocratique » de renforcement des inégalités et de l'exclusion, lesquelles seraient cependant masquées par des «pratiques de durabilité simulatives » et «d'auto-illusion». Cet article reconstruit les principales étapes argumentatives de cette approche et en discute les principaux présupposés, en particulier les notions d' « émancipation de deuxième ordre » et de « tournant postdemocratique ». Ces présupposés s'avèrent intenables aussi bien sur le plan historique que théorique. Ainsi, la tentative de Blühdorn d'appréhender les contradictions et les blocages des transformations écologiques ne mène-t-elle nulle part. La question qui se pose est alors de savoir quelles leçons on peut tirer de cet échec et quels desiderata de la recherche sociologique sur la durabilité et les transformations sociales en résultent.

Mots-clés Politique de non durabilité · Post-démocratie · Formes de sujet historiques · Émancipation de deuxième ordre $\cdot$ Politique simulative $\cdot$ Recherche sociologique sur la durabilité · Transformation socio-écologique

\section{Einleitung}

Nachhaltigkeitstransformationen sind seit gut zehn Jahren ein zentrales Thema auf der öffentlichen Agenda. In Deutschland wurde die Debatte zum einen durch die breit rezipierte Forderung des Wissenschaftlichen Beirates der Bundesregierung Globale Umweltveränderungen (WBGU 2011) nach einer „Großen Transformation“ zur Bewältigung der klimapolitischen Herausforderungen beflügelt, zum anderen durch die von der Weltfinanzkrise ausgelösten neuen kapitalismuskritischen Debatten und Bewegungen. Angesichts der dramatischen Beschleunigung des Klimawandels wie auch der sich immer weiter verschärfenden sozialen Ungleichheiten verknüpften sich beide Diskussionsstränge rasch zu einer intensiven, kontrovers geführten Debatte über die geeigneten Zielvisionen, Strategien und Ansatzpunkte einer beschleunigten sozial-ökologischen Transformation. Im Rahmen einer nun aufblühenden DegrowthBewegung wurden auch eine Fülle neuer praktischer Ansätze alternativen Wirtschaftens und Lebens entwickelt (vgl. u. a. Konzeptwerk Neue Ökonomie \& DFGKolleg Postwachstumsgesellschaften 2017). Ende 2015 einigte sich die Staatengemeinschaft in Paris auf ein Klimaabkommen, das in rechtlich verbindlicher Form die Begrenzung der globalen Erwärmung auf deutlich unter zwei Grad im Vergleich zum vorindustriellen Niveau verlangte. Die neue Dringlichkeit beschleunigter Nachhaltigkeitstransformationen wurde auch auf UN-Ebene durch die Verabschiedung der „Agenda 2030“ unterstrichen, die unter dem Titel „Transforming our World“ das Leitbild der Nachhaltigkeit in siebzehn vergleichsweise detaillierten ,Sustainable Development Goals“ konkretisierte. Die Fridays-for-Future-Bewegung konnte dann bis zum Beginn der Corona-Krise aufgrund einer breiten öffentlichen Unterstützung der Klimapolitik auf lokaler, nationaler und internationaler Ebene einen erheblichen Schub verleihen. Trotz dieser neuen Transformationsdebatten und Aktivitäten blieben die faktischen Veränderungen, zumindest auf der Ebene genereller Trends, 
zunächst gleichwohl sehr bescheiden. Die ergriffenen Maßnahmen ähnelten eher Trippelschritten und standen in einem immer größeren Kontrast zur Dramatik des fortschreitenden Klimawandels, des Artenverlusts, der Vermüllung der Meere etc., aber auch zur immer weiteren Verschärfung der globalen sozialen Ungleichheit.

Diese Situation löste auch in den Sozialwissenschaften eine neue Welle der Nachhaltigkeits- und Transformationsforschung aus (vgl. Adloff und Neckel 2019; Dörre et al. 2019; Schneidewind 2018; SONA 2021). Diese entwickelte sich in drei Strängen (Brand 2021). Während im Rahmen der ,transformativen Forschung“ meist in transdisziplinären, realexperimentellen Forschungssettings die Gestaltungsmöglichkeiten sozial-ökologischer Transformationen auf den verschiedenen Handlungsfeldern genauer ausgelotet wurden, wurden in wachstums- und kapitalismuskritischen Analysen sowohl die Grenzen und Widersprüchlichkeiten der etablierten, auf die Entwicklung einer Green Economy fokussierten Nachhaltigkeitsstrategien kritisiert als auch weitgespannte Transformationsvisionen entwickelt. Ein dritter, primär soziologischer Forschungsstrang richtete den Blick eher reflexiv-beobachtend auf die konkurrierenden gesellschaftlichen Nachhaltigkeitsdiskurse sowie auf die durch die faktischen Nachhaltigkeitstransformationen ausgelösten gesellschaftlichen Veränderungen, Widersprüche und Ambivalenzen.

In diesem Feld nehmen die Arbeiten von Ingolfur Blühdorn eine Sonderstellung ein. Blühdorn gilt spätestens seit dem Erscheinen seines Buches Simulative Demokratie (Blühdorn 2013) als einer der anregendsten Theoretiker der Nachhaltigkeitsund Demokratiedebatte. Blühdorns Zugang zur Nachhaltigkeitsthematik ist kein empirischer, seine Zeitdiagnosen folgen vielmehr einem großformatigen kritisch-modernisierungstheoretischen Ansatz. Er versteht seine theoretischen Analysen auch nicht als Beitrag zur transformativen Nachhaltigkeitsforschung, im Gegenteil. Seine Analyse tritt mit einem provokativen „metakritischen“ Anspruch auf, insofern sie die transformativen Ansätze der sozial- und politikwissenschaftlichen Nachhaltigkeitsforschung als Teil einer ,naiven Hoffnungserzählung“ kritisiert und ihnen Realitätsverweigerung vorwirft, weil sie an alten Emanzipationshoffnungen und an der Möglichkeit ,wirklicher“ Nachhaltigkeitstransformationen festhalten (Blühdorn 2018, S. 175 f.). Seine eigene These lautet dagegen, dass wir uns in Wirklichkeit alle in einem geschlossenen System der ,nachhaltigen Nicht-Nachhaltigkeit“ bewegen. Dieser Tatsache gelte es nüchtern ins Auge zu sehen.

Im vorliegenden Beitrag soll das Theoriedesign Ingolfur Blühdorns und seine grundlegenden Annahmen eingehender diskutiert werden. Im folgenden Abschnitt wird zunächst der Ausgangsbefund einer fast unübersehbaren, sich weltweit immer weiter auffächernden Vielfalt von Nachhaltigkeitsaktivitäten auf der einen, in zentralen Nachhaltigkeitsbereichen sich zugleich immer weiter verschärfenden Problemlagen auf der anderen Seite skizziert. Die Frage ist, wie sich dieser Widerspruch erklären lässt. Im dritten Abschnitt wird die Antwort Blühdorns, sein in den vergangenen zwei Jahrzehnten entwickelter Ansatz der „Politik der Nicht-Nachhaltigkeit“ im Detail rekonstruiert. Im vierten Abschnitt werden die zentralen Annahmen dieses Ansatzes näher diskutiert und kritisch hinterfragt; das sind vor allem die Konzepte der ,Emanzipation zweiter Ordnung“ und der ,postdemokratischen Wende“. Diese erweisen sich als wenig überzeugend. In einem fünften, abschließenden Abschnitt wird der Frage nachgegangen, was man aus dem Scheitern des Blühdorn'schen 
Erklärungsansatzes lernen kann und was sich daraus als zentrales Desiderat der soziologischen Nachhaltigkeits- und Transformationsforschung ergibt.

\section{Nachhaltige Nicht-Nachhaltigkeit - oder gebremste, widersprüchliche Transformationsdynamiken?}

Blühdorns zentrales Bezugsproblem ist der genannte Widerspruch zwischen der allgegenwärtigen Nachhaltigkeitsrhetorik und der faktischen Entwicklung, die für ihn eindeutig in eine entgegengesetzte Richtung geht. „Die Wende zur Nachhaltigkeit hat nicht bereits begonnen! Nichts deutet darauf hin, dass die Logik der Nicht-Nachhaltigkeit wirklich ernsthaft angeschlagen wäre. Eine Gesellschaft der Nachhaltigkeit ist - trotz neuer Klimabewegung, Grüner Wahlerfolge und vielfältiger ökologischer Modernisierungsprojekte - nirgends in Sicht“ (Blühdorn 2020a, S. 20f.). Im Gegenteil. Durch die üblichen „Aktivierungs- und Hoffnungserzählungen“ tragen wir auch noch zur Stabilisierung der etablierten Ordnung und ihrer Logik der NichtNachhaltigkeit bei (ebd.).

Angesichts früherer, im Rahmen der neuen sozialen Bewegungen der 1960er- und 1970er-Jahre geweckten Hoffnungen auf radikale, öko-egalitäre Gesellschaftstransformationen zeigt sich Blühdorn nach dreißig Jahren neoliberal geprägter Nachhaltigkeitspolitik grundlegend desillusioniert. Seitenverkehrt zur üblichen Nachhaltigkeitsdebatte fragt er deshalb nicht, wie Nachhaltigkeitsbarrieren beseitigt werden könnten, sondern danach, wie die paradoxe Konstellation der offenkundigen Nicht-Nachhaltigkeit unserer Lebensstile bei einem gleichzeitig hohen Stellenwert des Nachhaltigkeitsleitbilds in der Öffentlichkeit über Jahrzehnte hinweg stabilisiert werden konnte.

Die Diagnose der „nachhaltigen Nicht-Nachhaltigkeit“ (Blühdorn et al. 2020) wurde von Blühdorn bereits vor über zehn Jahren in ihren zentralen Elementen entwickelt und seither nur in verschiedenen Schritten aktualisiert (vgl. u. a. Blühdorn 2000, 2011, 2013, 2017, 2018). Die mit dem Aufschwung der Fridays-for-FutureBewegung gewachsene öffentliche Sensibilität für die Widersprüche zwischen der Dramatik des Klimawandels und der extremen Zögerlichkeit der offiziellen Klimapolitik hat ihr in den vergangenen Jahren aber eine erneute hohe Resonanz verschafft bis Corona alle anderen Themen erst einmal wieder von der Tagesordnung verdrängt hat. $\mathrm{Ob}$ dies längerfristig so bleibt (oder nicht) und wie die Corona-Krise die gesellschaftlichen Rahmenbedingungen für Nachhaltigkeitstransformationen insgesamt verändert, ist derzeit noch nicht genauer abzusehen; entsprechend kontrovers wird die Frage diskutiert (vgl. u. a. Brand 2020; Dörre 2020; Lessenich 2020; Rosa 2020). Im Rahmen der Klima- und der sozial-ökologischen Transformationsdebatten der vergangenen Jahre ist auf jeden Fall immer stärker ins öffentliche Bewusstsein gerückt, dass sich zentrale Problemtrends globaler Umweltveränderungen trotz der hohen gesellschaftlichen Resonanz des Nachhaltigkeitsleitbilds und trotz aller Nachhaltigkeitsaktivitäten weitgehend ungebrochen fortsetzen. So konnten zwar die Treibhausgasemissionen in den europäischen Ländern von 1990 bis 2020 durchschnittlich um etwa 25 Prozent gesenkt werden. Auf globaler Ebene steigen sie allerdings kontinuierlich weiter an, was aufgrund der beschleunigten industriellen 
Entwicklung Chinas, Indiens und vieler anderer Länder des globalen Südens auch nicht weiter verwunderlich ist. So sind sich die meisten Experten darin einig, dass Deutschland, wie fast alle anderen Länder, die selbstgesetzten Ziele zur Einhaltung der 2-Grad-Grenze deutlich verfehlen wird, wenn der Ausstieg aus der Nutzung fossiler Energien nicht extrem beschleunigt wird.

Der Klimawandel ist aber nur ein Problemfeld unter vielen anderen, wenn auch vielleicht das dringlichste. Das Modell der ,planetaren Grenzen“ (Steffen et al. 2015) verweist auf eine ganze Reihe anderer ökologischer Problembereiche, in denen sich die Entwicklung ebenfalls kritischen Grenzen nähert oder sie bereits überschritten hat. Der beschleunigte Verlust der Artenvielfalt, die Verschmutzung, Überfischung und Versauerung der Ozeane, die fortschreitende Bodendegradation und Entwaldung, die Süßwasserverknappung sowie viele andere Problemlagen verweisen auf die fortschreitende Übernutzung und Zerstörung von Ökosystemen und ihrer lebensnotwendigen Funktionen. Trotz der Ausweitung von Schutzgebieten an Land und im Meer, der Entwicklung nationaler Aktionspläne zur Umsetzung der Biodiversitätskonvention und der Ausweitung der Mittel für diese Zwecke schreitet der Verlust der Biodiversität in beispielloser Geschwindigkeit voran (CBD 2020).

Nimmt man nicht nur die Verschärfung globaler Umweltprobleme, sondern auch die in den „Sustainable Development Goals“ adressierten sozialen Probleme (Armut, Hunger, mangelnde Gesundheitsvorsorge, mangelnde Bildung, Geschlechterungerechtigkeit, soziale Ungleichheiten, Ausbeutung, Kriege etc.) sowie die Wechselwirkung beider Problemlagen hinzu, so verändert sich der Gesamteindruck nur unwesentlich. Im Bereich der Reduktion von extremer Armut, der Verringerung von Mütter- und Kindersterblichkeit, der Verbesserung des Zugangs zu Trinkwasser und sanitärer Versorgung, der Verbreitung von Primärbildung und der Geschlechtergleichstellung wurden in vielen Ländern des globalen Südens im Rahmen der ,Millennium Goals“ der Vereinten Nationen bis 2015 zwar bemerkenswerte Fortschritte erreicht; diese Erfolge waren regional und auf die gesamte Breite der „Sustainable Development Goals“ gesehen aber hochgradig selektiv (vgl. Köhler 2015; UN 2015). Zugleich hat sich in den vergangenen zehn Jahren die soziale Ungleichheit dramatisch weiter verschärft. Die Zahl der Milliardäre hat sich seit der Weltfinanzkrise nahezu verdoppelt. Weltweit besitzt der Club der Milliardäre - Oxfam zählte davon Ende letzten Jahres 2153 Personen - inzwischen ebenso viel wie 60 Prozent der Menschheit. Gleichzeitig können sich immer weniger Menschen aus extremer Armut befreien. Und Corona verschärft diese Ungleichheiten noch erheblich weiter (Oxfam 2019, 2021). Die Diagnose der ,nachhaltigen Nicht-Nachhaltigkeit“ hat also, zumindest pauschal betrachtet, sicher eine hohe Plausibilität.

Andererseits hat sich in den vergangenen 30 Jahren, seit dem Erdgipfel in Rio 1992, im Bereich der nachhaltigen Entwicklung auf lokaler, nationaler und transnationaler Ebene, im Bereich von Wirtschaft und Konsum, von Energieversorgung, Wohnen und Mobilität, von Landnutzung, Tierhaltung, Naturschutz etc. auch unendlich viel getan. Tausende von NGOs, Kommunen und Regierungen, zahllose Nachhaltigkeitsinitiativen, Wissenschaftler, Unternehmer und Konsumenten haben sich in den vergangenen zwei, drei Jahrzehnten weltweit für Nachhaltigkeitsziele engagiert. Insgesamt ergibt sich so ein äußerst widersprüchliches Bild. Auf der einen Seite besteht ein historisch unvergleichliches Wissen über Umwelt-, Klima- 
und Nachhaltigkeitsprobleme, über deren Risiko- und Katastrophenpotenziale, über die komplexen Verflechtungen lokaler Alltagspraktiken mit globalen ökologischen Entwicklungen und sozialen Ausbeutungsstrukturen. Das geht mit einer hohen Institutionalisierung von Nachhaltigkeitspolitiken auf den verschiedensten Themenfeldern und einem vergleichsweise hohen Engagement breiter Bevölkerungskreise einher. Auf der anderen Seite verschärfen sich zentrale Nachhaltigkeitsprobleme in einem kaum gebremsten Tempo, mit einem weltweit wachsenden Krisen- und Katastrophenpotenzial.

Die Frage ist, wie sich diese widersprüchlichen Befunde am besten erklären lassen. Blühdorns Erklärung gehört sicher zu den provokativsten Antworten, die sich derzeit finden lassen.

\section{3 ,Emanzipation zweiter Ordnung“, „Postdemokratie“ und „simulative Nachhaltigkeit“: Blühdorns ,,metakritische“" Analyse aktueller Entwicklungstrends}

Um Blühdorns Erklärung der zentralen Ursachen der ,,nachhaltigen Nicht-Nachhaltigkeit" zu rekonstruieren, verwende ich ein Analyseraster, das an anderer Stelle für den systematischen Vergleich gesellschaftstheoretischer Nachhaltigkeitsanalysen entwickelt wurde (Brand 2021). Es fächert zentrale analytische Dimensionen von Nachhaltigkeitstheorien zu dem Zweck auf, die zentralen Konzepte, impliziten Annahmen und Blindstellen des jeweiligen Theoriedesigns deutlich zu machen, um so die Transparenz sozialwissenschaftlicher Nachhaltigkeitsdebatten zu erhöhen. Unterschieden werden folgende Dimensionen:

- Zielsetzung und zentrales Anliegen der Studie.

- Untersuchungsgegenstand (thematischer Fokus) und sozial-räumliche Untersuchungsebene.

- Verständnis von Nachhaltigkeit (Zielvisionen und Bewertungsmaßstäbe).

- Problemdiagnose: Bestimmung der Ursachen von Nachhaltigkeitsproblemen, der als angemessen erachteten Lösungsstrategien und ihrer zentralen Blockaden.

- Disziplinärer Erklärungsansatz und gesellschaftstheoretische Analyse (Zeitdiagnose).

- Transformationsmodell: Theorie strukturellen gesellschaftlichen Wandels und Modell sozial-ökologischer Transformation.

- Bestimmung der globalen Kontextbedingungen von Nachhaltigkeitstransformationen (Megatrends, globale Krisendynamiken und Umbrüche), ihrer wechselseitigen Beeinflussung und Verknüpfung.

Wie lässt sich Blühdorns Ansatz anhand dieser Dimensionen aufschlüsseln?

(1) Blühdorns Ausgangspunkt und zentrales Bezugsproblem ist, wie eingangs bereits skizziert, der für ihn eklatante Widerspruch zwischen der allgegenwärtigen Nachhaltigkeitsrhetorik und der faktischen Entwicklung. Dass moderne Gesellschaften trotz aller institutionalisierten Umwelt- und Nachhaltigkeitspolitiken gerade „nicht nachhaltig“ sind, dass man nicht von einer „Gesellschaft der Nachhaltigkeit" (Neckel et al. 2018), sondern von einer ,Gesellschaft der Nicht-Nachhaltigkeit“" spre- 
chen müsse, erscheint Blühdorn und seinen Mitarbeiter*innen evident, nicht weiter begründungsbedürftig.

(2) Die apodiktische Feststellung, dass Gegenwartsgesellschaften definitiv nicht auf dem Weg in die Nachhaltigkeit sind, setzt eigentlich eine klare Definition von Nachhaltigkeit voraus, an der sich diese Behauptung messen lässt. Diese fehlt allerdings. Die eigenen Bewertungsmaßstäbe und die speziellen Nachhaltigkeitsprobleme, auf die sich Blühdorn bezieht, bleiben im Dunkeln - auch wenn ökologische Probleme offensichtlich die zentrale Referenz seiner Argumentation darstellen. Geht es ihm in der Tat aber nur um ökologische Nachhaltigkeit, und wenn ja, wie wird diese definiert? Geht es um eine Alternative zur ,Logik und Dynamik der unbestritten nicht-nachhaltigen modernen, kapitalistischen Konsumgesellschaft" (Blühdorn 2020b, S. 33)? Oder geht es um eine Alternative zu modernen, kapitalistischen Industriegesellschaften schlechthin, um eine Alternative zur „Logik der Vergrößerung, Vermarktlichung, Beschleunigung, Differenzierung, Individualisierung, Flexibilisierung, Singularisierung etc.“ (ebd., S. 31)? Wie sähen diese Alternativen dann aber aus?

Ganz generell gilt, dass die faktischen, widersprüchlichen Ansätze in Richtung nachhaltiger Entwicklung in ihrer Bedeutung umso mehr verblassen, je radikaler und utopischer das Gegenbild ,wirklicher“ Nachhaltigkeit imaginiert wird. Je nebulöser diese Gegenvisionen aber bleiben, je unklarer die Maßstäbe sind, an denen sich Fortschritte oder Rückschritte in Richtung Nachhaltigkeit bemessen lassen, desto mehr bewegt sich die Feststellung der ,unbestreitbaren“, ,definitiven“ NichtNachhaltigkeit aktueller Entwicklungen auf der Ebene suggestiver Beschwörungen.

(3) Dass das Konzept, die Vision und die Bewertungsmaßstäbe einer (substanziell) nachhaltigen Gesellschaft bei Blühdorn auch nicht in Ansätzen geklärt werden, hat vermutlich damit zu tun, dass weder die gesellschaftlichen Naturverhältnisse und die aus ihnen erwachsenden ökologischen Problemlagen noch die mit den bestehenden ökonomischen und politischen Verhältnissen verbundenen sozialen Problemlagen wie Hunger, Armut und Unterentwicklung in den Ländern des globalen Südens - die klassischen Bezugsprobleme des Konzepts nachhaltiger Entwicklung im Zentrum seiner Analyse stehen, sondern die Transformation der Demokratie und des emanzipatorischen Projekts der Moderne. Sich verschärfende ökologische, soziale und politische Probleme sind für ihn nur Nebenfolgen dieser politisch-kulturellen Transformation. Das erspart die Analyse der konkreten, sehr unterschiedlichen Problemsyndrome nicht-nachhaltiger Entwicklung, ihrer Ursachen und Lösungsmöglichkeiten. Da Blühdorn von der dauerhaften Nicht-Nachhaltigkeit demokratischer Politik und moderner Lebensstile als „unbestreitbarer“ Tatsache ausgeht, geht es ihm nur noch um die Frage, welche Stabilisierungsmechanismen das ,System der Nicht-Nachhaltigkeit“ dauerhaft am Leben erhalten. Folgt man dieser Ausgangsfrage, so rücken bei Blühdorn nicht die üblichen Verdächtigen wie Kapitalismus, Wirtschaftswachstum, Macht- und Interessenstrukturen, technische und infrastrukturelle Pfadabhängigkeiten etc. und deren komplexe Verknüpfungen ins Zentrum der Analyse, sondern ein spezifischer sozio-kultureller Komplex: die Veränderung der sozio-kulturellen Voraussetzungen moderner Demokratien und der damit verbundenen Subjektstrukturen. Das geht mit der sozial-räumlichen Verengung seines Blicks 
auf westliche Demokratien einher. Dass sich China und andere nicht-westliche Länder dieser Analyse nicht fügen, ist offenkundig.

(4) Wie wird dieser zentrale Bezugspunkt seiner gesellschaftstheoretischen Nachhaltigkeitsanalysen näher bestimmt? Für Blühdorn ist die sozio-kulturelle Basis des modernen Demokratieverständnisses das Konzept des vernünftigen, autonomen, mit unantastbarer Würde und unveräußerlichen Rechten ausgestatteten Individuums. Dieses Subjektverständnis gehe allerdings mit hohen Anforderungen an die Selbstdisziplin einher, wie sie Kant in seiner Pflichtethik, seinem kategorischen Imperativ und der Aufforderung, sich des eigenen Verstandes $\mathrm{zu}$ bedienen, formuliert habe. Diese sozio-kulturelle Basis, so die zentrale These, sei seit den neunziger Jahren des letzten Jahrhunderts einem grundlegenden Wandel unterworfen (Blühdorn 2000). Während die Vordenker der neuen sozialen Bewegungen Blühdorn zufolge noch fest in der Kant'schen Tradition verankert waren und im Anschluss an die Kritische Theorie individuelle Autonomie und Integrität der Natur noch als zwei Seiten einer Medaille verstanden, habe sich seither durch die Radikalisierung kultureller Modernisierungsprozesse ein grundlegender Wandel der Wert-, Subjekt- und Identitätsstrukturen vollzogen. Im Kern dieses sozio-kulturellen Strukturwandels steht das, was Blühdorn, paradox zugespitzt, als „Emanzipation zweiter Ordnung“ bezeichnet (Blühdorn 2013, S. 144 ff.; 2020c, S. 65 ff.). Während die „Emanzipation erster Ordnung" auf die Ausweitung der Möglichkeiten der Selbstbestimmung und Selbstorganisation zielte, gehe es in der „Emanzipation zweiter Ordnung“, in der Radikalisierung individueller Selbstverwirklichungsansprüche, ,um den Auszug aus der selbst erstrittenen Mündigkeit“ (Blühdorn 2013, S. 144). Gemeinwohlorientierte Verpflichtungen und gesellschaftliche Verantwortung werden zur „Befreiung von moralischen und intellektuellen Selbstüberforderungen“" an Institutionen und professionelle Dienstleister delegiert, um ,die sich lebensweltlich jeweils bietenden Handlungs- und Selbstverwirklichungsoptionen“ unbeschwert ausnutzen zu können (ebd., S. 145).

Im Rahmen dieses sozio-kulturellen Transformationsprozesses sei die Norm des autonomen, identischen Subjekts als „Zentralkategorie der Demokratie“ erodiert (ebd., S. $128 \mathrm{ff}$.). Das autonome Subjekt - verstanden ,as (a) unitary, consistent, principled, stable and identical, and (b) composed of innate qualities of character and inner values as opposed to anything external, material, ephemeral and superficial“" (Blühdorn 2017, S. $51 \mathrm{f}$.) - werde zum einen zunehmend vom Markt durchdrungen und vereinnahmt. Konsumistische Lebensstile, die bei Marcuse und anderen Vertretern der Kritischen Theorie noch als Inbegriff der Entfremdung galten, werden zum neuen Kern subjektiver Identitäten. Zum anderen werde das Ideal des in sich stabilen identischen Subjekts abgelöst ,,vom Ideal des vielschichtigen und flexiblen [...] Subjekts“ (Blühdorn 2013, S. 132), wie es von den Theoretikern der Postmoderne als neue Subjektform der „flüchtigen Moderne“ beschrieben wurde (Baumann 2003). Die Verflüssigung der Identitäten werde dabei nicht nur durch die neoliberale Vermarktlichung des gesellschaftlichen Lebens, sondern auch durch die neuen digitalen Medien vorangetrieben, die der Image-Produktion, dem Ego-Marketing und der eigenen Medien-Resonanz einen zentralen Stellenwert für das Selbstverständnis und die eigene Identität verleihen (Blühdorn 2017, S. 52). Diese neuen konsumtiven Subjekt- und Identitätsideale schaffen ihre „eigenen kategorischen Imperative“, die 
auch neuer Wege bedürfen, um ihnen gerecht zu werden. Die funktionale Antwort darauf beschreibt Blühdorn mit den Begriffen der ,ppostökologischen Wende“ und der „Politik der Nicht-Nachhaltigkeit“" (ebd.).

Aus dem beschleunigten Umbau der Subjektivitätsnorm erwächst, so Blühdorn, zum einen ein Formwandel der Demokratie. Mit der Auflösung der Norm des autonomen, vernunftbasierten, politisch verantwortlich handelnden Subjekts zugunsten eines auf konsumistische Selbstverwirklichung bezogenen postmodernen Subjektverständnisses zerfalle auch die normative Grundlage moderner partizipativer Demokratien. Mit dieser ,postdemokratischen Wende“ habe sich das emanzipative „demokratische Projekt [...] schlicht überlebt“; es könne auch nicht mehr reanimiert werden (Blühdorn 2013, S. 150). Zugleich werden die Ansprüche auf demokratische Repräsentation, Partizipation und Legitimation aber nicht aufgegeben. Das ergibt ein für „Postdemokratien“ typisches Paradox (ebd., S. 158ff.): die widersprüchliche Entwicklung zwischen der mit der Erosion des autonomen Subjekts und der neoliberalen Vermarktlichung der Gesellschaft verbundenen Entpolitisierung des Politischen auf der einen Seite (Privatisierung der Politik, Anpassungszwänge an den globalen Markt, Expertenregime, New Public Management, subjektive Delegierung von Verantwortung, Befreiung von egalitären Gemeinwohlverpflichtungen etc.) und der gleichzeitigen „maßlosen Aufblähung“ von Freiheits-, Subjektivierungs-, Selbstbestimmungs- und Partizipationsansprüchen auf der anderen Seite. Die Lösung für dieses ,postdemokratische Paradox“ sieht Blühdorn in der ,simulativen Politik", die die demokratischen Normen, von denen die Bürger sich eigentlich verabschiedet haben und denen auch keine realen Politiken mehr entsprechen, durch Diskurse, Narrative und Selbstbeschreibungen, aber auch durch Aktivierung von Beteiligung, durch die Bereitstellung partizipativer Erlebnis- und Handlungsräume inszeniere (ebd., S. 178). Simulative Politik beruhe dabei auf einer Art ,neuem Gesellschaftsvertrag zwischen den verschiedensten gesellschaftlichen Akteuren“, der „die etablierten gesellschaftlichen Konfliktlinien überbrückt und nicht so sehr auf eine Täuschung der Massen durch die herrschenden Eliten zielt, sondern eher auf eine Art gesellschaftliche Selbstillusionierung, zu der die verschiedensten Akteure und Teile der Gesellschaft beitragen“" (ebd., S. 232).

Diese kritische demokratietheoretische Argumentationsstruktur überträgt Blühdorn auch auf die Nachhaltigkeitsthematik. Das emanzipative Projekt der politischen Ökologie werde im Rahmen der „Emanzipation zweiter Ordnung“ durch einen ,post-ecologist turn" abgelöst, der öko-egalitäre Visionen in die realpolitischen Fahrwasser grüner Politik lenkt (Blühdorn 2000; Hervorh. K.-W. B.). Diese postökologische Wende ,erweist sich als ein effektiver Beschleuniger des materiellen Konsums und ist wenig geeignet für jede Form der die Mehrheit betreffenden Einschränkung von Rechten und materiellen Ansprüchen, wie sie für eine wirklich effektive Politik der Nachhaltigkeit wohl unerlässlich wäre" (Blühdorn 2013, S. 237 f.). Stattdessen erhebe das flexible, markt- und konsumorientierte Subjekt die „Nicht-Nachhaltigkeit gerade zum Prinzip“ (ebd., S. 245). Stetig wachsende Bedürfnisse, konsumistische Lebensstile und Selbstverwirklichungsansprüche gelten zunehmend als ,unverhandelbar“. Lösungsstrategien werden nicht mehr in der notwendigen Begrenzung ,entfesselter Freiheiten“, sondern in der Logik des Markts, 
in ökonomischen Anreizen und technologischen Innovationen gesehen (ökologische Modernisierung, Green Economy).

Parallel zum ,postdemokratischen Paradox“ diagnostiziert Blühdorn so ein ,postökologisches Paradox“. Es bestehe darin, dass moderne Gesellschaften heute zwar ein umfassendes Bewusstsein der Nachhaltigkeitsprobleme und der drohenden ökologischen Katastrophen haben, ,unter dem Druck der [...] radikalisierten Selbstverwirklichungsansprüche [aber] fester denn je entschlossen [seien], die etablierten Werte und Strukturen [...] mit allen zur Verfügung stehenden Mitteln zu verteidigen“" (ebd., S. 250 f.). Angesichts sich zuspitzender ökologischer Nachhaltigkeitsprobleme werde die Aufrechterhaltung ,unserer Freiheit, unserer Werte, unseres Lebensstils“ zur obersten Priorität. Damit wächst die Notwendigkeit der ,umfassenden gesellschaftlichen Externalisierung sozialer und ökologischer Kosten“ sowie der ,innergesellschaftlichen Umverteilung und Exklusion“, um die konsumbasierte Form des modernen Lebens aufrechterhalten zu können (Blühdorn 2020c, S. 109). Eine an den Wertpräferenzen der Bürger orientierte Politik werde zwangsläufig zur „Politik der Nicht-Nachhaltigkeit“ (Blühdorn 2011; 2013, S. 258 ff.). Im Namen der „,bedrohten Mehrheit" vollziehe sich stillschweigend eine Transformation der Demokratie „,von einem Instrument zur Sicherung universaler Rechte, Freiheiten und Integrität - auch für die Natur - und zur möglichst gerechten Verteilung von gesellschaftlichem Wohlstand in ein Instrument zur [...] Legitimation von wachsender Ungleichheit und Exklusion“ (Blühdorn 2018, S. 172).

Da die Widersprüche zwischen Nachhaltigkeitspostulaten und nicht nachhaltigen Lebensstilen aber durchaus noch wahrgenommen werden, bedarf es entsprechender Kommunikationsformen und Handlungsarenen, in denen die Verpflichtung auf Werte der Nachhaltigkeit zum Ausdruck gebracht werden kann, ohne die Errungenschaften der „Emanzipation zweiter Ordnung“ zu gefährden (Blühdorn 2017, S. 57). Das ist die Ebene der simulativen Nachhaltigkeitsdiskurse und Nachhaltigkeitspraktiken, der ,Praktiken der gesellschaftlichen Selbstillusionierung“ (Blühdorn 2013, S. 256). Nicht nur die etablierten Formen der Nachhaltigkeitskommunikation, sondern auch alle Reformansätze und Initiativen, alle Klima- und Postwachstumsbewegungen, alle Narrationen einer „Großen Transformation“ bewegen sich aus Blühdorns Sicht immer nur in diesem geschlossenen Zirkel der Selbstillusionierung. Lokale Initiativen neuer, nachhaltigerer Produktions- und Lebensformen ermöglichen zwar die praktische Erprobung alternativer Werte und bieten individuelle Ausdrucksmöglichkeiten für ökologisches und soziales Commitment. Sie sind aber in Makrostrukturen der Nicht-Nachhaltigkeit eingebettet und bleiben deshalb ,,purely experimental and experiential“, ohne weitere strukturelle Konsequenzen (Blühdorn 2017, S. 57).

(5) Wenn alle transformativen Nachhaltigkeitsbemühungen aber ohnehin nur Praktiken der Selbstillusionierung, nur Stabilisierungsmechanismen sich immer weiter verschärfender Exklusionspolitiken zur Verteidigung der eigenen nicht-nachhaltigen Lebensstile sind, dann bedarf es offensichtlich auch keiner speziellen Transformationstheorie mehr. In Blühdorns Arbeiten wird die Frage, wie sich ein grundlegender sozial-ökologischer Strukturwandel überhaupt vollziehen und mithilfe welcher Strategien und Ansatzpunkte er beschleunigt werden könnte, denn auch völlig ausgeblendet. Abgesehen von der - aufgrund des Formwandels der Demokratie ohnehin illusorischen - Forderung nach einer strikten „Begrenzung und Regulierung“ 
der durch die Emanzipation zweiter Ordnung entfesselten Freiheiten moderner Konsumgesellschaften (Blühdorn 2020c, S. 114), findet sich bei Blühdorn kein weiterer Hinweis auf Dynamiken, Ansatzpunkte und Gestaltungsmöglichkeiten einer solchen tiefgreifenden gesellschaftlichen Transformation. Die durch naturwüchsige sozio-kulturelle Modernisierungsdynamiken historisch entstandene Konstellation moderner „Konsumentendemokratien“ wird als festgezurrtes systemisches Gefüge der Nicht-Nachhaltigkeit verstanden, als ,schwarzes Loch“, in dessen Sog alle transformativen Ansätze, Bewegungen und Initiativen verschwinden. Aus der Sicht Blühdorns ändert daran auch die aktuelle Corona-Krise wenig; sie verfestige nur regressive, ausgrenzende Tendenzen. Das verleiht seinen Analysen den Charakter des Tragischen, Ausweglosen. Ökologische Katastrophen und die kontinuierliche Verschärfung sozialer Ausgrenzung und Polarisierung erscheinen unaufhaltsam. Grundlegende Veränderungen werden in dieser Erzählung nur noch als postkatastrophische Entwicklung denkbar.

Umso stärker stilisiert sich Blühdorn in der Rolle des unbestechlichen Beobachters, als ,metakritischer“ Aufklärer, der verhängnisvolle Tendenzen aufzeigen muss, auch wenn sie sich nicht mehr stoppen lassen (Blühdorn 2013, S. 279f.; 2020d, S. $313 \mathrm{ff}$.). Das geht mit einer scharfen elitären Abgrenzung von der ,transformativen“ Forschung einher, die mit ihren illusionären Hoffnungserzählungen und der „Weigerung, aus dem Emanzipationsparadigma auszubrechen und anzuerkennen, dass die Emanzipationslogik längst im Gleichklang mit der Wachstums- und Expansionslogik steht“", nur „Teil eines stillen Gesellschaftsvertrags für das ,Weiter-So “" wird (Blühdorn 2018, S. 176). Die für die sozialwissenschaftliche Nachhaltigkeitsforschung einzig angemessene Haltung sei dagegen, sich aller aktivistischen Illusionen zu entziehen und der sich vollziehenden tragischen Entwicklung nüchtern ins Auge zu blicken.

\section{4 ,Nachhaltige Nicht-Nachhaltigkeit“" - oder doch nur Blindstellen der Analyse?}

Wie ist diese pointierte Diagnose zu bewerten? Wie überzeugend sind ihre zentralen Konzepte? Lenkt sie das Augenmerk auf zentrale Ursachen der global sich weiter verschärfenden Trends nicht-nachhaltiger Entwicklung, oder blockiert sie eher deren Verständnis?

Auf drei zentrale Blindstellen der Analyse Blühdorns wurde bereits verwiesen. Das betrifft erstens die fehlende Definition des Konzepts oder der Vision von Nachhaltigkeit, an der die konkreten Nachhaltigkeitspolitiken und Nachhaltigkeitspraktiken gemessen werden und auf die sich die Diagnose stützt, dass die ,Wende zur Nachhaltigkeit" definitiv noch nicht begonnen hat. Damit eng verbunden ist die zweite Blindstelle einer fehlenden Präzisierung der Nachhaltigkeitsprobleme, auf die sich Blühdorns Diagnose bezieht. Der Klimawandel hat andere konkrete Ursachen als Gewässerverschmutzung, Armut oder Geschlechterungleichheit. Erst wenn die Maßstäbe von Nachhaltigkeit sowie die zentralen Bezugsprobleme und deren Ursachen näher bestimmt werden, lässt sich sinnvoll über die Angemessenheit oder Nichtangemessenheit von Nachhaltigkeitsstrategien sprechen. Eine dritte zentrale 
Blindstelle ist das Fehlen eines Transformationsmodells. Wenn kein Begriff davon besteht, wie sich tiefgreifende sozial-ökologische Transformationen vollziehen und wie sie gestaltet werden könnten, bleibt auch offen, ob ,wirkliche“ Veränderungen bereits auf den Weg gebracht wurden oder sich alles nur im Rahmen rein ,simulativer" Praktiken bewegt. Die Ergebnisse allein, etwa die weitere globale Verschärfung der Klimaproblematik, sagen darüber ja noch wenig aus. Wirkungen und Nebenfolgen bestimmter Interventionsmaßnahmen zeigen sich aufgrund der Eigendynamiken ökologischer Systemdynamiken immer erst zeitversetzt.

Damit hat sich Blühdorns Diagnose der ,nachhaltigen Nicht-Nachhaltigkeit“ gegen empirische Kritik bereits weitgehend immunisiert, bleibt so allerdings auch ziemlich beliebig. Eine höhere Plausibilität kann Blühdorns Diagnose nur durch die Überzeugungskraft seiner inhaltlichen Argumente gewinnen. Diese sollen deshalb im Folgenden eingehender diskutiert werden. Im Vordergrund stehen dabei die für ihn zentralen Thesen der „Emanzipation zweiter Ordnung“ und der ,postdemokratischen Wende“.

\subsection{Das ,,autonome, identische Subjekt" - Träger von Demokratie und Nachhaltigkeit?}

Blühdorn schließt die Relevanz von Interessenstrukturen, Machtverhältnissen oder kapitalistischen Verwertungsimperativen als Erklärungsfaktoren für die Nicht-Nachhaltigkeit moderner Gesellschaften zwar nicht grundsätzlich aus; sie sind seiner Ansicht nach aber völlig unzureichend, um die Persistenz des - aus seiner Sicht geschlossenen, sich selbst legitimierenden Systems der Politik der Nicht-Nachhaltigkeit zu erklären. Wie wir gesehen haben, ist der für ihn entscheidende Faktor die sozio-kulturelle Veränderungsdynamik von Subjekt- und Identitätsstrukturen, die seit den neunziger Jahren sich vollziehende „Emanzipation zweiter Ordnung“. Das setzt als historische Kontrastfolie die Existenz nachhaltigerer Subjektstrukturen vor den 1990er-Jahren voraus. Wie plausibel ist diese Annahme?

Blühdorns Bezugspunkt ist nun allerdings gar keine näher bestimmte historische Subjektform moderner Industriegesellschaften (etwa des liberalen, des organisierten oder des wohlfahrtsstaatlichen Kapitalismus), sondern das normative Modell des ,autonomen“, durch den Bezug auf die „Vernunft“ sich selbst begrenzenden Subjekts. Dieses Subjektmodell bildet die Basis des modernen natur- und vernunftrechtlich begründeten Staatsverständnisses. „Vernunft“ avanciert in der Neuzeit zur zentralen Kategorie, auf die sich nicht nur die wissenschaftliche Erklärung und technische Gestaltung der Welt, sondern auch die Legitimation der politischen und gesellschaftlichen Ordnung stützt. Die Problematik dieses Bezugspunkts im Rahmen der Blühdorn'schen Analyse ist aber, dass das Modell des ,,autonomen Subjekts“ als eine historische Subjektform (miss)verstanden wird. Inwieweit ein auf Vernunftkriterien basierendes Gemeinwesen demokratisch organisiert sein kann, wird im 18. und 19. Jahrhundert selbst in bildungsbürgerlichen Kreisen noch sehr skeptisch beurteilt. Kant war selbst ein Republikaner, der von der Demokratie, anders als vom Rechtsstaat, eher eine Gefährdung vernunftbasierter gemeinwohlorientierter Entscheidungen befürchtete. De facto sind Aufklärung und Bildung nur auf kleine privilegierte Teile der männlichen Bevölkerung begrenzt. Und nur wer über Eigentum 
verfügt, gilt gemeinhin als Bürger. Damit wird zunächst der größte Teil der Bevölkerung (Frauen, Arme, Landarbeiter, Proletarier, Sklaven) aus dem Kosmos der politischen Bürger, der ,,autonomen“, politisch zur Selbstbestimmung berechtigten Individuen ausgegrenzt. Der darin implizierte Widerspruch zum universellen Postulat der Menschenrechte speist seither einen Großteil der gesellschaftlichen Kämpfe um Anerkennung und politische Teilhabe.

Dass das moderne, emanzipative Demokratiemodell konstitutiv mit der Kant'schen Norm des autonomen, vernunftbasierten Subjekts verknüpft ist, gilt somit nur sehr begrenzt. Diese These verkennt die heterogenen Stränge modernen demokratischen Denkens und die realen Entwicklungsdynamiken liberaler Demokratien. Deren Legitimität beruht nicht primär auf dem kategorischen Vernunftprinzip Kants. Die naturrechtlich begründete Idee der unveräußerlichen Menschenrechte gewinnt in Europa vielmehr in zwei Theoriesträngen politisches Gewicht: zum einen in einer marktliberalen Variante, wie sie in der Locke'schen Theorie formuliert und nicht nur für die amerikanische Unabhängigkeitsbewegung, sondern für westliche Verfassungen ganz generell konstitutiv wurde; zum anderen in der basisdemokratischen Variante des Rousseau'schen Demokratiemodells, das für die Französische Revolution und für nachfolgende revolutionäre Demokratiebewegungen eine zentrale Bedeutung gewann, mit der postulierten Identität von Einzel- und Gemeinwille aber immer auch die Gefahr des Totalitarismus in sich barg. Zwischen diesen beiden Demokratiemodellen besteht seit jeher ein ausgeprägtes Spannungsverhältnis. Demokratien legitimieren sich im Normalfall, in den stabileren Phasen gesellschaftlicher Entwicklung, nicht durch Entscheidungsverfahren, in denen der „Allgemeinwille“ oder die „Vernunft“ der Bürger ,,authentisch“ zum Ausdruck gebracht wird, sondern durch Mehrheitsentscheidungen im Rahmen eines repräsentativen Politikmodells, durch Freiheits-, Rechtsstaats- und Sicherheitsgarantieren sowie durch (halbwegs) funktionierende öffentliche Versorgungs- und Dienstleistungen. Basisdemokratische emanzipatorische Leitbilder gewinnen dagegen immer in gesellschaftlichen Aufbruchs-, Reform- und Revolutionsphasen an Bedeutung.

Was Blühdorn zu einem grundlegenden historischen Wandel von der „Emanzipation erster Ordnung“ zur „Emanzipation zweiter Ordnung“ stilisiert, lässt sich so zum einen, wesentlich weniger dramatisch, als Übergang von der emanzipativen Aufbruchsphase der neuen sozialen Bewegungen hin zu einer neuen Normalitätsphase des seit den neunziger Jahren sich etablierenden neoliberalen Ordnungsmodells begreifen. In diesem Rahmen erlangen besitzindividualistische Orientierungen, der Rückzug ins Private und die politische Delegation von Verantwortlichkeiten wieder ein stärkeres Gewicht. Solche zyklischen, mit langen ökonomischen Wellen und der periodischen Restrukturierung gesellschaftlicher Regulationsmodelle verbundenen Pendelbewegungen von emphatischen Aufbruchs- zu neuen Normalitätsphasen prägen seit dem 19. Jahrhundert die Entwicklung aller westlichen Gesellschaften (vgl. Brand 2018). Das Wechselspiel von öffentlichem Engagement und privatem Rückzug ist somit nichts Neues, sondern der Normalfall liberaler, marktwirtschaftlich organisierter Demokratien (Hirschman 1982).

Emanzipative Aufbruchsphasen sind dabei keineswegs intrinsisch mit der Idee des ,autonomen, identischen Subjekts“ verknüpft. In Europa wurde die Demokratie im späten 19. und frühen 20. Jahrhundert primär von der Arbeiterbewegung erkämpft, 
deren Trägergruppen ganz andere Identitäten und Selbstverständnisse aufwiesen als das Bildungsbürgertum. Postkoloniale Befreiungsbewegungen oder schwarze Bürgerrechtsbewegungen stützten sich zwar auf die Prinzipien universeller Menschenrechte; ihre Identitäten waren aber ebenfalls nicht die aufgeklärter, autonomer, bildungsbürgerlicher Individuen. Auch die neuen sozialen Bewegungen speisten sich aus äußerst heterogenen kulturellen Orientierungen und stützten sich auf ein breites Spektrum von Protestakteuren aus unterschiedlichen sozialen Milieus (Brand et al. 1986; Kriesi et al. 1995). Die bereits gegen Ende des 19. Jahrhunderts einsetzende schrittweise Dekonstruktion des klassisch-bürgerlichen Subjektverständnisses machte denn auch deutlich, dass das bürgerliche Selbstverständnis des autonomen Subjekts sowohl die Umsetzung der universellen Menschenrechte als auch die Entwicklung eines neuen, nachhaltigeren Naturverhältnisses lange Zeit eher blockiert hat. Das rationale bürgerliche Subjekt ist historisch aufs Engste mit der Kolonialisierung der Welt, aber auch mit dem Projekt der technischen Beherrschung und der ökonomischen Ausbeutung der Natur verknüpft. Erst die postmoderne, postkoloniale und feministische Kritik der „Metaerzählungen der Moderne“, insbesondere des linearen, eurozentrischen Fortschrittsdenkens, öffnete den Blick für die Ambivalenz und Kontingenz geschichtlicher Entwicklungen und ermöglichte es, die radikale Pluralität der Welt anzuerkennen (vgl. Conrad und Randeria 2002; Katz und Winterfeld 2006; Latour 1998; Merchant 1987; Welsch 1990). Insgesamt erweist sich der von Blühdorn gewählte Kant'sche Bezugspunkt des ,autonomen, identischen Subjekts“ so weder als eine entscheidende sozio-kulturelle Voraussetzung für das emanzipative Demokratieverständnis noch als Grundlage für einen besonders nachhaltigen Umgang mit der Natur.

\subsection{Die ,Emanzipation zweiter Ordnung“ - Schlüssel zum Verständnis der ,nachhaltigen Nicht-Nachhaltigkeit"?}

Bricht damit das eine Standbein der These der „Emanzipation zweiter Ordnung“ weg, so ist die Frage, inwieweit sie vom zweiten Standbein, der Analyse der veränderten, postmodernen Subjektstrukturen noch getragen werden kann. Klar ist, dass die erwähnten periodischen Pendelbewegungen von politischen Aufbruchs- hin zu privatistischen Rückzugsphasen nur einen Teil der von Blühdorn und anderen beobachteten politisch-kulturellen Veränderungen seit den neunziger Jahren erklären können, auf jeden Fall nicht das historisch Neue daran. Worin besteht dies? Zum einen kommen die kulturrevolutionären Effekte der neuen sozialen Bewegungen nun langsam auch im Alltag breiter Bevölkerungsschichten zum Tragen (vgl. Brand 2010). Individualisierungsdynamiken und neue hedonistische Orientierungen verändern Lebenseinstellungen, Geschlechterverhältnisse, soziale Beziehungen, Freizeit und Konsum. Mit dem Zusammenbruch des Ostblocks, der Stabilisierung des neoliberalen Ordnungsmodells und den beschleunigten Globalisierungsdynamiken verändern sich aber auch die politischen Wahrnehmungsmuster sowie die Organisationsstrukturen von Wirtschaft, Gesellschaft und Politik. Damit bilden sich auch neue Lebensstile und Subjektformen aus. Ein stärker individualisiertes, mobiles, marktorientiertes, an kreativer und konsumtiver Selbstverwirklichung orientiertes Subjekt wird zum gesellschaftlichen Leitmodell. Dass sich diese neuen Konsumiden- 
titäten an einem sehr flüssigen, sich ständig verschiebenden Spektrum symbolisch hoch aufgeladener Dinge, Markenartikel, Dienstleistungen, Wohnformen, Esskulturen, Freizeitpraktiken, Urlaubsorte etc. bilden, gilt Blühdorn nun als Beleg für das „haltlos“ gewordene, „flüssige“ Individuum, das zu einer vernünftigen, ökologisch oder sozial gebotenen Selbstbegrenzung nicht mehr in der Lage sei.

$\mathrm{Ob}$ es sinnvoll ist, die Besonderheiten der sich herausbildenden neuen Subjektformen nur oder vorranging über die Konsumintensität der neuen Lebensstile zu beschreiben, lässt sich sicher hinterfragen. Kapitalismuskritische Analysen kommen zu einer etwas anderen Akzentuierung. Hier sind es die mit der Deregulierung der Finanzmärkte, der Entfesselung wirtschaftlicher Globalisierungsdynamiken, dem Abbau sozialstaatlicher Sicherungssysteme und der umfassenden Vermarktlichung des gesellschaftlichen Lebens verbundenen Veränderungen des Arbeits- und Alltagslebens, die den neuen Subjektformen ihr spezifisches Gepräge geben. Die veränderten Rahmenbedingungen, politischen Diskurse und Alltagerfahrungen machen das ,,selbstverantwortliche“, im permanenten Wettbewerb mit anderen stehende ,unternehmerische Subjekt“ zur neuen Norm (Bröckling 2007; Lessenich 2008). Das schafft nicht nur in beruflicher Hinsicht einen neuen Zwang zur „Selbstoptimierung“, es schafft auch auf der privaten Ebene einen neuen Zwang zur Erlebnisoptimierung und zur Inszenierung des eigenen ,singulären“ Lebensstils (Reckwitz 2017). Dieser Modus, sich seiner eigenen Identität zu versichern, wird durch die rasante Verbreitung der digitalen sozialen Medien seit den 2010er-Jahren noch einmal erheblich verstärkt.

Auch auf politischer Ebene führen die wirtschaftlichen Globalisierungsdynamiken und die neoliberale Transformation des öffentlichen Lebens zu erheblichen Veränderungen. Privatisierung, Public-Private Partnerships und New Public Management gelten nun als effizienteste Formen der öffentlichen Verwaltung. Hierarchische Steuerungsmodelle verwandeln sich in dezentrale Formen der Governance, in der auch partizipative Verfahren und NGOs ein größeres Gewicht erlangen. Hier fügen sich die Mitte der neunziger Jahre aufblühenden lokalen Agenda-21-Prozesse und deliberativen Politikmodelle als zeitgenössische Reformvarianten problemlos ein (Zilleßen et al. 1993). Aus linker Perspektive wird dagegen die Transformation westlicher Demokratien in „Postdemokratien“ beklagt (Crouch 2008; Rancière 1996). Grundlegendere politische Auseinandersetzungen verlieren danach zugunsten eines als ,alternativlos“ erachteten Managements der wirtschaftlichen Globalisierungsfolgen an Bedeutung. Das Politische werde nur noch symbolisch inszeniert; das schaffe Frustrationen und fördere eine generelle Entpolitisierung. Die damit verbundene Forderung nach einer partizipativen Revitalisierung der Demokratie erscheint Blühdorn nun zwar als sympathisch, historisch aber als überholt, weil sich die Subjektstrukturen in irreversibler Weise vom ,autonomen, identischen Subjekt“ hin zum fluiden, an ,entfesselten“ Selbstverwirklichungsinteressen orientierten Subjekt verschoben hätten.

Nun liegt dieser Annahme, wie wir bereits im letzten Abschnitt gesehen haben, ein klassischer Fehlschluss zugrunde: die Verwechslung der präskriptiven mit der deskriptiven Ebene. Dem stilisierten Idealtypus des für die moderne Staatstheorie konstitutiven, durch die Orientierung am vernünftigen Allgemeininteresse sich selbst beschränkenden Individuums wird der empirische Typus des an konsumti- 
ver Selbstverwirklichung orientierten postmodernen Individuums gegenübergestellt. Verglichen werden könnten aber nur entweder die normativen Modelle oder die historisch-empirischen Varianten beider Subjekt- und Identitätsformen. Im ersten Fall müsste dem autonomen bürgerlichen Subjekt der moralische Idealtypus des - in der Nachhaltigkeitsdebatte ja vielfach beschworenen - ,verantwortlichen“ oder ,nachhaltigen Konsumenten“ gegenübergestellt werden. Auch von diesem wird ein am allgemeinen Interesse der Menschheit, ein an den Prinzipien der intra- und intergenerationalen Gerechtigkeit orientiertes Verhalten gefordert, das die Berücksichtigung komplexer ökologischer und sozialer Zusammenhänge verlangt, meist entgegen allen kurzfristigen rationalen Eigeninteressen (z. B. an niedrigen Preisen). Das wird in vielen Fällen, zumindest partiell, auch versucht - und durch soziale Distinktionspraktiken gesellschaftlich stabilisiert (vgl. Neckel 2018, 2020). Im zweiten Fall ergäbe sich für beide historische Typen ein hochgradig widersprüchliches Verhältnis zwischen moralischen Selbstverständnissen auf der einen, etablierten Alltagspraktiken und Lebensformen auf der anderen Seite.

Blühdorns These der „Emanzipation zweiter Ordnung“ führt aber auch deshalb in die Irre, weil sie eine homogene postmoderne Subjektstruktur unterstellt, aus der dann wiederum ein einziger zentraler politischer Imperativ postmoderner „Konsumentendemokratien“ abgeleitet wird. Selbst hegemoniale Subjektformen sind aber keine homogene Einheit, sondern „hybride Subjekte“, wie Andreas Reckwitz in einer historisch breit angelegten kultursoziologischen Analyse moderner Subjektkulturen gezeigt hat (Reckwitz 2006). Sie stellen eine spezifische Kombination unterschiedlicher, durchaus widersprüchlicher kultureller Codes und Sinnelemente dar, die im Rahmen bestimmter historischer Formationen der Moderne eine Antwort auf die Frage geben, was Subjekte ausmacht. Diese Subjektformen werden immer im Kontext von Alltagspraktiken hervorgebracht. Für Reckwitz sind es vor allem drei Praxiskomplexe, in denen Subjekte geformt werden: ökonomische Praktiken der Arbeit, Praktiken persönlicher und intimer Beziehungen sowie die „Technologien des Selbst“, zu denen er auch den Umgang mit Medien sowie - seit dem 20. Jahrhundert - die Praktiken des Konsums zählt (ebd., S. 16f.).

Reckwitz hat in diesem Sinne die Entfaltung und Transformation dreier hegemonialer Subjektformen der Moderne rekonstruiert. In Abgrenzung vom Adel versucht „die bürgerliche Moderne des 18. und 19. Jahrhunderts die Form des moralisch-souveränen, respektablen Subjekts verbindlich zu machen; die organisierte Moderne der 1920er bis 1970er Jahre produziert als Normalform das [an einem sozialtechnischen Ingenieurmodell trainierte] extrovertierte Angestelltensubjekt; die Postmoderne von den 1980er Jahren bis in die Gegenwart entwickelt das Modell der kreativ-konsumtorischen Subjektivität“ (ebd., S. 15). Die drei identifizierten modernen Subjektformen, die ihre Legitimität jeweils durch die Distanzierung von der vorhergehenden Subjektordnung gewinnen, bilden ihrerseits jeweils gegenhegemoniale subkulturelle Subjektformen aus: die Romantik schafft in der bürgerlichen Moderne das Gegenmodell des ,expressiven Individualsubjekts“, der ästhetische Modernismus der organisierten Moderne das ästhetische „Avantgardesubjekt“ und die kulturrevolutionäre „counter culture“ der 1960er- und 1970er-Jahre das „gegenkulturelle Subjekt“ der Postmoderne (ebd.). Erst in diesen spannungsreichen hybriden Verhältnissen bildet sich für Reckwitz die für die jeweilige Zeit typische Subjektordnung aus. 
Auffällig ist bei Reckwitz sein ästhetisch-kultureller Fokus. Würden nicht nur die für die jeweiligen Phasen typischen antihegemonialen Ausprägungen der „,Künstlerkritik“, sondern auch der „Sozialkritik“ mitberücksichtigt (vgl. Boltanski und Chiapello 2003), so würden nicht nur die verschiedenen Formationen der Moderne, sondern auch ihre jeweiligen, in Spannung zueinander stehenden Subjektkulturen eine etwas andere Gestalt annehmen. Auch die Bestimmung und zeitliche Zuordnung der verschiedenen gegenhegemonialen Bewegungen und Subjektkulturen ließe sich diskutieren. So blühten die gegenkulturellen, gesellschaftskritischen Strömungen und Bewegungen der 1960er- und 1970er-Jahre nicht zu Beginn der Postmoderne, sondern am Ende des langgezogenen Wirtschaftswachstums der Nachkriegsjahrzehnte als Reaktion auf das „fordistische“, an funktionaler Planung, Normierung und Standardisierung orientierte Gesellschafts- und Subjektmodell auf und trugen erheblich zu dessen Erosion bei; sie sind nicht das ,gegenkulturelle Subjekt der Postmoderne“, sondern dessen Wegbereiter. In einer stärker auf Arbeit und Wirtschaft fokussierten Analyse vertreten auch Boltanski und Chiapello die These, dass die 68er-Bewegung einem ,neuen Geist des Kapitalismus“, einer neuen Motivations- und Rechtfertigungsstruktur kapitalistischer Gesellschaften, den Weg bereitet hat. Die Frage ist somit immer, wie oppositionelle gegenkulturelle Bewegungen, die in der Phase der Erschöpfung etablierter Gesellschafts- oder Regulationsmodelle an Gewicht gewinnen, in nicht-intendierten Modernisierungseffekten die Gestalt der neuen Ordnungsmodelle mitprägen (vgl. Kungl 2021).

Was die Beschreibung des postmodernen Subjekts betrifft, so unterscheidet sich diese bei Reckwitz nicht grundsätzlich von der Blühdorns, auch wenn Reckwitz' Analyse die kulturell-kreativen Aspekte postmoderner Subjekte stärker betont. Die für unsere Zwecke entscheidende Differenz zwischen Reckwitz' und Blühdorns Analyse liegt allerdings darin, dass Reckwitz das Subjekt der Postmoderne weder als homogene Einheit noch als Endprodukt einer historischen Entwicklung begreift. Während Blühdorn pauschal vom konsumtiven postmodernen Subjekt spricht, ist für Reckwitz klar, dass die jeweils hegemonialen Subjektformen immer von bestimmten Klassen und sozialen Milieus getragen werden. So verortet er das postmoderne, ,kreativ-konsumtorische Subjekt“" in den akademischen Mittelschichtmilieus der neuen urbanen, mit dem kulturellen Kapitalismus verbundenen „creative classes“. Daneben existieren aber immer auch andere Klassen und Milieus mit gesellschaftlich peripheren Subjektformen. In der postindustriellen Gesellschaft der „Spätmoderne“ sind dies für Reckwitz neben einer kleinen Oberklasse der Superreichen vor allem das neue, unter prekären Bedingungen lebende Dienstleistungsproletariat sowie die alte Mittelklasse, die in der Polarisierung zwischen der neuen Mittel- und der neuen Unterklasse zunehmend zerrieben werde (Reckwitz 2019). Historisch dominante Subjektformen sind so nicht nur selbst immer hybride Arrangements heterogener, widersprüchlicher kultureller Codes, sie sind auch Teil eines gesellschaftlich sehr viel differenzierteren Gefüges von klassen- und milieuspezifischen Identitäten und Subjektformen. Daraus ergibt sich im Rahmen repräsentativer Demokratien dann auch kein einheitlicher, ,unverhandelbarer“ politischer Imperativ der Sicherung konsumtiver, nicht-nachhaltiger Lebensstile. Die Erwartungen an Sicherheit und Wohlstand werden vielmehr, je nach Lebenslage, Klassen- und Milieuzugehörigkeit, sehr unterschiedlich definiert und mit jeweils anderen Weltbildern, Narrationen und politischen 
Aktionsprogrammen verknüpft. Das gilt auch für die Antworten auf Umwelt- und Nachhaltigkeitsprobleme.

\subsection{Die ,postdemokratische Wende“ - Formwandel der Demokratie?}

Insgesamt spricht somit nichts für die These, dass postmoderne Lebensstile und Selbstverwirklichungsansprüche eine ,postdemokratische Wende“ erzwingen, die die Demokratie zwangsläufig in ein politisches Instrument zur Aufrechterhaltung des „Systems der Nicht-Nachhaltigkeit“ und der sozialen Exklusion verwandelt. Weder macht die historische Gegenüberstellung des vernunftbasierten, bürgerlichautonomen (= nachhaltigen) und des an konsumtiver Selbstverwirklichung orientierten postmodernen (= nicht-nachhaltigen) Subjekts Sinn, noch sind postmoderne, neoliberal strukturierte Gesellschaften sozial und kulturell so homogen, dass sich daraus eindeutige politische Handlungsimperative ergeben. Die Dynamiken neoliberaler Vergesellschaftung haben zwar neue Arbeits-, Lebens- und Subjektformen geschaffen, die Heterogenität und Gegensätzlichkeit der Lebenslagen und politischkulturellen Orientierungen aber nicht beseitigt. Die postmoderne „Gesellschaft der Singularitäten“ (Reckwitz 2017) zeichnet sich vielmehr durch eine wachsende kulturelle Diversität von Lebensformen und Gruppenidentitäten, aber auch durch wachsende soziale Polarisierungen aus.

All das schließt aus, dass moderne Demokratien aus Legitimationserfordernissen einem einzigen „unverhandelbaren“ Imperativ der Gewährleistung konsumbasierter, nicht-nachhaltiger Lebensstile folgen müssen. Selbst wenn die Sicherung des jeweils erreichten gesellschaftlichen Wohlstandsniveaus aus Legitimations- und Machterhaltungsgründen ein zentrales Ziel demokratischer Regierungen darstellt, so steht dieses Ziel nicht nur in Konkurrenz zu anderen Staatszielen (wie die Corona-Politik zeigt); es ist auch hochgradig umstritten, was dieses Ziel angesichts wachsender ökologischer, gesundheitlicher und sozialer Problemlagen, angesichts des digitalen Umbruchs von Wirtschaft und Gesellschaft und angesichts sich verschärfender internationaler Hegemonialkonflikte impliziert und mithilfe welcher Strategien diese Krisen bewältigt werden sollen. Nirgendwo ist ein Konsens über diese Frage in Sicht. Abwehr und Exklusion sind nicht die einzig möglichen Antworten.

Die „,radikalisierten konsumtiven Selbstverwirklichungsansprüche“ haben in den vergangenen Jahren auch niemand daran gehindert, sich in neuen emanzipativen Bewegungen zu engagieren. Mit der Krise des Neoliberalismus gewannen nicht nur neue globalisierungs- und kapitalismuskritische Bewegungen an Bedeutung (etwa die Occupy-Bewegung); auch neue Demokratiebewegungen (wie im ,,arabischen Frühling“) und neue Klimagerechtigkeits- und Degrowth-Bewegungen blühten auf und nicht nur im Westen, sondern weltweit. Die konsumbasierten, postmodernen Lebensstile verhinderten auch keineswegs das Aufleben eines neuen Feminismus und einer neuen Antirassismus-Bewegung. All diese Bewegungen speisen sich aus der Einforderung universeller, durch das Nachhaltigkeitsleitbild neu gerahmter Menschenrechte. Ganz offensichtlich behalten demokratische und ökologische Werte ihre Mobilisierungskraft über den historischen Wandel von Subjektstrukturen hinweg. Nichts - außer fragwürdigen theoretischen Konstrukten - spricht dafür, dass dies alles nur simulative Aktivitäten, symbolische Garnierung einer ,,unverhandel- 
bar“ auf die Imperative konsumbasierter Lebensstile festgelegten „Postdemokratie“ sind. Es sind vielmehr Auseinandersetzungen und Kämpfe um die Restrukturierung eines neuen, nachhaltigeren gesellschaftlichen Ordnungsmodells.

Damit wird keineswegs behauptet, dass sich in der Postmoderne das Verständnis von und die Beziehung zur Politik nicht verändert hätten. Das haben sie sehr wohl. Die beschleunigten Individualisierungsprozesse des späten 20. Jahrhunderts haben den Zerfall traditioneller Gemeinschaften und politischer Milieus vorangetrieben. Das geht mit dem Bindungsverlust von Großorganisationen und Volksparteien einher. Auch die Akzeptanzbereitschaft von Mehrheitsentscheidungen hat sich verringert. Das gilt insbesondere für gesellschaftlich stark umstrittene Themen. Dissens, Opposition oder Widerstand bei (meist lokalen) Konfliktthemen, die als existenziell erachtet werden, lassen sich durch Parteien und Mehrheitsentscheidungen kaum mehr einbinden. Die Schwelle für themenspezifische Mobilisierungen, ob für „Mut“oder „Wutbürger“, ist erheblich gesunken. Generell ist an die Stelle langfristiger politischer Bindungen und Organisationsmitgliedschaften ein temporäres, an spezifischen Problemen orientiertes, auch von Moden und Stimmungslagen geprägtes gesellschaftliches Engagement getreten (vgl. Enquete-Kommission 2002). Zugleich gewinnen neue partikulare Formen des Sozialen und Politischen, die Ausdifferenzierung in Szenen, Subkulturen und Communities, in sub- und identitätspolitische Gemeinschaften an Bedeutung (Reckwitz 2017, S. 10). Die zentrale Rolle des Marketings in postmodernen, neoliberalen Gesellschaften verschafft darüber hinaus der symbolischen Inszenierung von Politik und ihren Vertretern ein immer größeres Gewicht. Die neuen sozialen Medien verstärken diesen Trend noch weiter. Auch das liefert aber keinen hinreichenden Grund für die Annahme, die Politik hätte sich zwangsläufig in eine „Politik der Nicht-Nachhaltigkeit“" verwandelt. Die veränderten Formen politischer Kultur und die erhöhte Notwendigkeit der symbolischen Inszenierung von Politik betreffen jede politische Position und jede Art öffentlicher Mobilisierung gleichermaßen. Akzeptanz muss heute immer aktiv beschafft werden, das gilt für Politiken der Nicht-Nachhaltigkeit wie der Nachhaltigkeit.

Auch die weltweit beobachtbare Krise liberaler Demokratien, die Verbreitung autoritärer Regime und rechtspopulistischer Bewegungen (vgl. Geiselberger 2016), hat wenig mit der Herausbildung postmoderner Subjektstrukturen zu tun. Sie ist vielmehr, so die hier vertretene Gegenthese, mit der weltweiten Krise des neoliberalen Regulationsmodells verknüpft. Anders als in den 1970er- und 1980er-Jahren, als der Zerfall des „fordistischen“ Regulationsmodells das westlich-liberale Ordnungsmodell als solches nicht in Frage stellte, ist die Erosion des neoliberalen Regulationsmodells heute allerdings mit einer umfassenden Hegemonialkrise des westlichen Ordnungsmodells verbunden. Die durch die frühe Industrialisierung Europas ermöglichte, im Rahmen der kolonialen Eroberungen des 19. Jahrhunderts auch politischmilitärisch abgesicherte Vormacht des Westens kommt heute an ihr Ende. Damit werden ökonomisch, politisch und kulturell die Karten neu gemischt. Überkommene Machtgefüge, Abhängigkeiten und Hegemonien werden infrage gestellt; China gewinnt eine wachsende Bedeutung im Weltsystem (vgl. Schmalz 2018). Die historische Kopplung der Verbreitung moderner liberaler Demokratien mit der Verbreitung des fossilen Industrialisierungs- und Wachstumsmodells (und seiner ökologischen Folgeprobleme) auf der einen und dem Nationalstaatsprinzip auf der anderen Seite 
wirft zusätzliche Probleme auf. Ähnliches gilt für die digitale Transformation der herkömmlichen Formen demokratischer Öffentlichkeit und Beteiligung. Ohne eine grundlegende Erneuerung und organisatorische Restrukturierung liberaler Demokratien wird es kaum gelingen, ihre Ausstrahlungskraft als emanzipative, zugleich aber auch effiziente, zur Lösung zentraler gesellschaftlicher Probleme geeignete staatliche Organisationsform zu bewahren.

\section{Nachhaltigkeitstransformationen verstehen}

Die hier angedeuteten alternativen Erklärungsperspektiven der Krise moderner Demokratien müssen nicht unbedingt geteilt werden; sie sollten nur verdeutlichen, dass es zu Blühdorns Problemdeutungen sehr wohl Alternativen gibt. Die Thesen der „Emanzipation zweiter Ordnung“ und der ,postdemokratischen Wende“ haben sich auf jeden Fall als wenig überzeugend erwiesen. Gibt es aber kein funktional geschlossenes, wegen der vermeintlichen Unverhandelbarkeit konsumtiver Lebensstile zu Strukturreformen unfähiges System der ,nachhaltigen Nicht-Nachhaltigkeit“, so bedarf es anderer Erklärungen für die strukturellen Blockaden, Widersprüchlichkeiten und Ambivalenzen von Nachhaltigkeitsprozessen. Das von Daniel Hausknost (2020) im Umfeld Blühdorns entwickelte, staatstheoretisch begründete Theorem der „gläsernen Decke der Transformation“ schafft in dieser Hinsicht keine Abhilfe. Dieses Konzept unterstellt, dass die Legitimationsimperative demokratischer Staaten die Fixierung auf Wachstumspolitik und die Stabilisierung des einmal erreichten Lebensstandards erzwingen. Staatliche Nachhaltigkeitspolitik sei ,,auf lebensweltund wachstumskompatible Politiken“ festgelegt (ebd., S. 157). Sie könne so nie die Grenze zwischen ,,partikularem Umweltschutz“ und ,systemischer Nachhaltigkeitspolitik“ überschreiten (ebd., S. 146f.).

Ist die Dekarbonisierung moderner Gesellschaften, der Ersatz fossiler durch erneuerbare Energiesysteme aber nur ,,partikularer Umweltschutz“? Hat die Dekarbonisierung nicht viel weiter reichende sozial-ökologische Folgen (die von manchen mit der neolithischen und der industriellen Revolution verglichen werden)? Ist der Einstieg in die stoffliche Kreislaufwirtschaft, so bescheiden diese Ansätze bisher auch noch sein mögen, nur „Umweltschutz“? Bewegt sich die von vielen Städten aus klimapolitischen Gründen forcierte Entwicklung nachhaltigerer Mobilitätssysteme, nachhaltigerer Bau- und Siedlungsformen unterhalb der ,gläsernen Decke“ und wann, bei welchen Maßnahmen wäre sie überschritten? Wo liegt die ,gläserne Decke“ für die angestrebte „Agrarwende“? Liegt sie bei 20, 30 oder 50 Prozent Ökolandbau? Hausknosts Argument hängt so genauso in der Luft wie Blühdorns Argument. Es bewegt sich innerhalb desselben funktionalistisch geschlossenen Argumentationsmodells, das auf der Vorannahme beruht, dass ,,wirkliche“ Nachhaltigkeit im Rahmen der gegebenen Verhältnisse definitiv nicht möglich ist. Wie sich gezeigt hat, ist die dafür gebotene inhaltliche Erklärung aber mehr als brüchig.

Wir stehen nach dieser - zugegebenermaßen etwas aufwändigen - Auseinandersetzung mit Blühdorns Thesen somit wieder mit leeren Händen da, wenn wir den widersprüchlichen Befund der seit den neunziger Jahren in den meisten Ländern der Welt zu beobachtenden verstärkten Nachhaltigkeitsaktivitäten auf der einen, 
der ungebrochen fortwirkenden oder sich weiter verstärkenden nicht-nachhaltigen Trends (zumindest im Bereich globaler Umweltveränderungen) auf der anderen Seite erklären wollen (vgl. Brand 2017). Dieses Ergebnis kontrastiert scharf mit der lautstarken Forderung Blühdorns nach „leistungsfähigen Theoriemodellen“, die die „,naiven Hoffnungserzählungen“ der transformativen Nachhaltigkeitsforschung ersetzen sollten. Die von ihm selbst gebotenen Erklärungen fallen weit hinter diesen Anspruch zurück.

Blühdorn folgt einem eindimensionalen, funktionalistischen Erklärungsmodell, das die kulturellen Antinomien, die Heterogenität und Widersprüchlichkeit, den Herrschafts- und Kontrollcharakter der Moderne und die daraus sich immer wieder neu entwickelnden Konflikte und gesellschaftlichen Kämpfe unterschlägt. Er thematisiert Entwicklung als linearen Prozess der Entfaltung und Radikalisierung bestimmter Aspekte der Moderne (Emanzipation und Selbstverwirklichung) und operiert mit einem eigentümlichen, idealistisch verzerrten Demokratiebegriff als Kontrastfolie für aktuelle Entwicklungen. Er blendet die konkreten Entstehungszusammenhänge von Nachhaltigkeitsproblemen aus der Analyse aus, suggeriert aber doch, dass sie alle, ganz pauschal, in den konsumintensiven, postmodernen Lebensstilen begründet seien; die „Begrenzung überbordender Ansprüche auf Freiheit und konsumbasierte Selbstverwirklichung“" sei deshalb auch die adäquate Antwort auf global sich verschärfende Nachhaltigkeitsprobleme (Blühdorn 2019). Was das konkret bedeutet, wie sich die verschiedenen Nachhaltigkeitsprobleme (z. B. postfossile Transformationen) mit einer solchen „Begrenzungsstrategie“ lösen ließen und mithilfe welcher Wirtschafts- und Politikmodelle das ggf. durchgesetzt werden könnte, bleibt aber völlig offen. ${ }^{1}$ Seine Analyse ist darüber hinaus auf sozio-kulturelle Entwicklungsdynamiken westlicher Gesellschaften fokussiert, als ob sich daraus allein die globalen Dynamiken nicht-nachhaltiger Entwicklung ableiten ließen.

Was lässt sich daraus für problem- und gegenstandsadäquate Nachhaltigkeits- und Transformationsanalysen lernen?

Zunächst wäre es hilfreich, die eingangs des dritten Abschnitts skizzierten zentralen Analysedimensionen sozialwissenschaftlicher Nachhaltigkeitstheorien systematischer zu reflektieren, um typische Blindstellen der jeweiligen Analysen identifizieren und ihre Transparenz erhöhen zu können. Was die inhaltliche Analyse von Nachhaltigkeitsproblemen und ihrer gesellschaftlichen Bearbeitung betrifft, so besteht ein breiter Konsens in der einschlägigen Forschung, dass monokausale Erklärungen und Ansatzpunkte in jedem Fall zu kurz greifen. Die Entwicklung moderner Gesellschaften und der damit verknüpften Naturverhältnisse wird - auch bei einer Dominanz bestimmter Organisationsprinzipien (z. B. kapitalistischer Markt- und Verwertungsprinzipien) - nie durch ökonomische, technische, politische, soziale oder kulturelle Entwicklungslogiken allein vorangetrieben, sondern immer nur durch ihre komplexen, widersprüchlichen Wechselwirkungen und die daraus erwachsenden Nebenfol-

\footnotetext{
1 Zu Recht kritisiert Neckel (2020, S. 87 und 97 ff.) die von Blühdorn vertretenen autoritären Kontrollvisionen als demokratiefeindlich. Diese Kontrollvisionen stünden nicht nur in einem grundlegenden Widerspruch zu den selbst postulierten emanzipativen, basisdemokratischen Wertorientierungen; Blühdorns Argumentation stehe auch in einer deutlichen Kontinuität zur konservativen Kritik an der ,Anspruchsinflation“ in den 1970er-Jahren.
} 
gen. Für das Verständnis von Nachhaltigkeitsproblemen bedarf es darüber hinaus immer interdisziplinärer Forschungsansätze, die die Verknüpfung unterschiedlicher Entwicklungs- und Problemdimensionen in den Blick nehmen. Erst daraus ergeben sich adäquate Ansatzpunkte für sozial-ökologische Transformationen.

Was die gesellschaftliche Seite dieser Transformationsanalysen betrifft, so setzen sie ein Verständnis des Zusammenhangs von Strukturentwicklung und Handlungsprozessen voraus. Strukturelle oder systemische Entwicklungen folgen in modernen, hochdifferenzierten Gesellschaften zwar immer gewissen Eigenlogiken; diese gewinnen ihre materielle Wirksamkeit aber nur über millionenfaches individuelles Handeln, im Medium institutioneller Routinen und Alltagspraktiken. Entwicklungen auf der Struktur- und der Handlungsebene vollziehen sich dabei zwar nicht synchron, aber doch immer in rekursiven Prozessen (Bourdieu 1982; Giddens 1988). Das lässt sich theoretisch unterschiedlich beschreiben. Für eine handlungsnahe Analyse (nicht-)nachhaltiger Entwicklungen haben sich in den vergangenen Jahren (um die materielle Dimension erweiterte) praxistheoretische Ansätze als besonders geeignet erwiesen, da sie neben kulturellen Orientierungen, sozialen Rahmenbedingungen und habituellen Handlungskompetenzen auch die materiellen Infrastrukturen sowie die für die jeweiligen Praxiszusammenhänge relevanten naturalen Umwelten systematisch in die Analyse mit einbeziehen können (vgl. u. a. Brand 2011; Reckwitz 2003; Schatzki 1996; Shove 2003; Shove et al. 2007). Das eröffnet die Möglichkeit, nicht nur die komplexen sozio-materiellen Handlungsverflechtungen und Abhängigkeiten, sondern auch die möglichen Ansatzpunkte für die Veränderung von sozialen Praktiken genauer bestimmen zu können.

Dass Strukturen und Handlungen immer nur zwei Seiten einer Medaille, eines rekursiv sich stabilisierenden oder verändernden Prozesses sind, sagt allerdings noch wenig über die treibenden Faktoren (nicht-)nachhaltiger Entwicklungen aus. Der hohe Energie-, Material- und Naturverbrauch unserer heutigen Lebensstile (Ernährung, Mobilität, Wohnen, Kleidung, Körperpflege, Reinigung, Medienkonsum, Freizeitverhalten etc.) ist ja nicht intendiert, nicht der Zweck der jeweiligen Alltagspraktiken, sondern eine zunächst wenig beachtete, erst im Kontext der Umwelt- und Nachhaltigkeitsdebatte der vergangenen Jahrzehnte zunehmend bewusst gewordene Implikation dieser Alltagspraktiken. Deren Vermeidung wird allerdings nur im Rahmen spezieller, explizit ökologisch orientierter Lebensstile und Distinktionspraktiken zum direkten Handlungsziel. Die Nachhaltigkeitsimplikationen der einzelnen Konsumhandlungen $\mathrm{zu}$ bedenken, ist im Normalfall ohne extreme Vereinfachung hochkomplexer Sachverhalte auch kaum einlösbar (eindeutige Label oder klare Entscheidungen: Bio, kein/wenig Fleisch, keine/wenig Flugreisen, ÖPNV statt Auto etc.). Der Appell an den ,verantwortlichen Konsumenten“ kann ohnehin nur dann greifen, wenn nachhaltigere Alternativen bereitstehen, um die jeweiligen Handlungsziele zu erreichen.

Das macht deutlich, dass ein Großteil der Alltagspraktiken überhaupt nicht frei gewählt, sondern Teil eines sozio-materiellen Handlungsgefüges ist, das durch das komplexe Zusammenwirken technischer, ökonomischer, politischer und kultureller Entwicklungen und die darin implizierten Formen der Naturnutzung historisch entstanden ist. Postmoderne Lebensstile sind ein konstitutives Element hoch industrialisierter und urbanisierter, global vernetzter, durch die Dynamiken kapitalistischer 
Märkte vorangetriebener, durch fossile Energien befeuerter und durch technische Infrastrukturen in ihrem Funktionsbestand gesicherter Wohlstandsgesellschaften. Unsere Formen des Wohnens, der Mobilität, der Ernährung, der Energienutzung, der Berufstätigkeit etc. sind durch diese Rahmenbedingungen entscheidend geprägt. Sie sind durch individuelle Konsumentscheidungen deshalb nur begrenzt modifizierbar. Die Nachhaltigkeitsdebatte der vergangenen Jahrzehnte hat so auch hinreichende Belege dafür geliefert, dass der Versuch, Konsumenten zum Dreh- und Angelpunkt nachhaltiger Entwicklung zu machen, entschieden zu kurz greift; Konsum ist überwiegend „systemischer Konsum“ (vgl. u. a. Blättel-Mink 2016; Brand 2008; Grunwald 2012; Mock 2020).

Umgekehrt lassen sich dann aber auch die zentralen Blockaden von Nachhaltigkeitstransformationen nicht primär den einzelnen Individuen, ihren Wertorientierungen und Selbstverwirklichungsbedürfnissen, der „Unverhandelbarkeit“ ihrer Konsum- oder Lebensstile zurechnen. Ob Häuser als Energieschleuder oder Energiesparhäuser gebaut werden, ob die Energie durch fossile oder postfossile Energieträger gewonnen wird, ob motorisierte Fahrzeuge mit Benzin oder Batterien angetrieben werden, ob die Landwirtschaft konventionell oder ökologisch betrieben wird, macht für den Komfort individuellen Lebens kaum einen Unterschied, ist klimapolitisch aber hochrelevant. Diese Alternativen ergeben sich aber so gut wie nie aus individuellen Konsumentscheidungen - auch wenn Pioniermodelle eine zentrale Voraussetzung für die Möglichkeit entsprechender Energie-, Mobilitäts- oder Agrarwenden darstellen. Sie sind vielmehr immer Folge des komplexen Zusammenwirkens neuer Problemwahrnehmungen, politischer Entscheidungen, technischer Entwicklungen und veränderter ökonomischer Rahmenbedingungen. Auf das Auto zugeschnittene Infrastrukturen und Siedlungsformen verwandeln sich nicht von selbst, evolutionär, in eine postfossile Mobilitätsstruktur; das gelingt auch nicht von heute auf morgen. Infrastrukturell verankerte Wirtschafts- und Lebensweisen weisen eine hohe Pfadabhängigkeit auf und lassen sich nur in längerfristigen Rhythmen verändern. Das Gleiche gilt für die Energiegewinnung und Energieversorgung. Um fossile Energien durch regenerative Energien zu ersetzen, bedarf es klarer staatlicher Vorgaben, massiver Förderungen und Anreize - und länger terminierter Ausstiege aus der fossilen Energienutzung. Erfolgreiche „Wenden“ setzen, um soziale Akzeptanz zu finden, auch eine soziale Abfederung und neue Berufsperspektiven für die negativ betroffenen Bevölkerungsgruppen voraus. All das wird von heftigen gesellschaftlichen Auseinandersetzungen begleitet, deren Ausgang immer offen ist.

Es gibt somit nicht den einen Dreh- und Angelpunkt der fortwirkenden NichtNachhaltigkeit unserer Wirtschafts- und Lebensformen. Nachhaltigkeitstransformationen stoßen vielmehr auf eine Vielzahl von Blockaden (vgl. Berg 2020). All das ist gleichwohl kein Grund für die Annahme, dass Nachhaltigkeitstransformationen definitiv nicht stattfinden. Transformative Bemühungen bewegen sich auf sehr unterschiedlichen Ebenen und verfolgen sehr unterschiedliche Zielvisionen. Sie setzen auch nie pauschal, sondern immer nur an spezifischen, öffentlich präsenten, medial hinreichend dramatisierten Problemen an. Sie stützen sich auf bestimmte Problemdeutungen und darauf bezogene Lösungsstrategien - und verfolgen meist auch nur solche Strategien, die (mit möglichst geringen Kosten) auch als machbar erscheinen. Das gilt für persönliche Veränderungen, das Engagement in Organisationen 
oder auf der politischen Ebene. Alle praktischen Transformationsansätze bewegen sich nicht nur in einem Feld hoher Ungewissheit, was Problemdeutungen sowie die Wirkungen und Nebeneffekte der jeweiligen Lösungsstrategien betrifft, sie bewegen sich auch in einem hoch kontroversen, von Deutungskämpfen, Interessenkonflikten und Machtstrukturen geprägten Feld. Einfache Reforminstrumente, wie z. B. das Erneuerbare-Energien-Gesetz, können dabei erstaunliche Transformationseffekte erzielen; andere können aber auch völlig versanden. Umgekehrt können auch radikale Veränderungsansätze gesellschaftlich auflaufen.

Das schafft insgesamt einen von hohen Unsicherheiten, Konflikten und Machtkämpfen, von infrastrukturellen, institutionellen und kulturellen Pfadabhängigkeiten geprägten, durch immer wieder andere Themen, Problemlagen und Krisen überlagerten Transformationskontext. Es verleiht Nachhaltigkeitstransformationen ihren spezifisch widersprüchlichen, von Kompromissen und Blockaden, aber auch von überraschenden - oft mit dramatischen Ereignissen verbundenen - Durchbrüchen und der partiellen Verstetigung einzelner Transformationspfade bestimmten Charakter. Die Wahrscheinlichkeit, dass dieser auf den verschiedensten Ebenen laufende, gleichwohl aber äußerst zähe sozial-ökologische Transformationsprozess mit der wachsenden Dramatik globaler Umweltveränderungen und den damit verbundenen sozialen Krisen und Verwerfungen nicht Schritt hält, ist sicher nicht gering zu veranschlagen. Offen ist auch, inwieweit ökologische und soziale Nachhaltigkeitsziele gleichzeitig zielgerichtet verfolgt werden können oder ob dies jeweils anderer Transformationsstrategien bedarf. Was in dieser Situation Not täte und von Soziologen und anderen Sozialwissenschaftlern erwartet werden könnte, ist, das Verständnis von Nachhaltigkeitstransformationen weiter zu klären.

Das zentrale Problem dabei ist, dass eine umfassende, tiefgreifende, leitbildgesteuerte Transformation gesellschaftlicher Basisstrukturen und Naturverhältnisse historisch völlig präzedenzlos ist, die Sozialwissenschaften deshalb auch über keinen adäquaten Begriff eines solchen Transformationstypus verfügen (vgl. Reißig 2009). Politische Revolutionstheorien sind hier wenig ergiebig. Der um die Jahrtausendwende florierenden, modernisierungstheoretisch inspirierten Transformationsforschung ging es primär um die Analyse des gelingenden oder scheiternden Übergangs postsozialistischer Staaten in kapitalistische, liberal verfasste Gesellschaften, nicht aber um die Transformation zentraler Strukturprinzipien moderner Industriegesellschaften als solcher (vgl. Kollmorgen et al. 2015). Die vorliegenden komplexitätstheoretischen Modelle der sozial-ökologischen Innovations- und Transformationsforschung machen ihrerseits zwar deutlich, wie sich Veränderungen sozio-technischer Regime im Spannungsfeld von Nischeninnovationen und Megatrends vollziehen und politisch gestalten lassen (vgl. Grin et al. 2010; Kemp et al. 2007; Rückert-John und Schäfer 2017). Unterschlagen werden aber meist die sozialen Dimensionen dieser Transformationsprozesse, die Machtgefüge, Ungleichheitsstrukturen und zentralen gesellschaftlichen Konflikte, die mit solchen Transformationsprozessen immer verbunden sind. Und sie lassen völlig im Unklaren, wie Armut, Hunger, hohe Kinderund Müttersterblichkeit, soziale Ungleichheiten und Kriege beseitigt und ob diese unterschiedlichen Nachhaltigkeitsziele überhaupt, außer im Rahmen normativer Zielkataloge wie den „Sustainable Development Goals“, systematisch miteinander verknüpft werden können. Auf die Frage, wie ,große“ sozial-ökologische Trans- 
formationen erfolgreich und ohne dramatische soziale und politische Verwerfungen bewerkstelligt werden können, bieten die etablierten Forschungsansätze und Gesellschaftstheorien bisher noch wenig Antworten (vgl. Adler 2016; Dörre et al. 2019; Görg et al. 2017; Kropp 2019; Schneidewind 2018; WBGU 2011). Differenzierte gesellschaftstheoretische Debatten über den neuen, experimentellen Typus struktureller Nachhaltigkeitstransformationen, seine möglichen Verlaufsformen, Entwicklungsdynamiken und Risiken, stellen somit ein zentrales Desiderat der sozialwissenschaftlichen Nachhaltigkeitsforschung dar.

Funding Open Access funding enabled and organized by Projekt DEAL.

Open Access Dieser Artikel wird unter der Creative Commons Namensnennung 4.0 International Lizenz veröffentlicht, welche die Nutzung, Vervielfältigung, Bearbeitung, Verbreitung und Wiedergabe in jeglichem Medium und Format erlaubt, sofern Sie den/die ursprünglichen Autor(en) und die Quelle ordnungsgemäß nennen, einen Link zur Creative Commons Lizenz beifügen und angeben, ob Änderungen vorgenommen wurden.

Die in diesem Artikel enthaltenen Bilder und sonstiges Drittmaterial unterliegen ebenfalls der genannten Creative Commons Lizenz, sofern sich aus der Abbildungslegende nichts anderes ergibt. Sofern das betreffende Material nicht unter der genannten Creative Commons Lizenz steht und die betreffende Handlung nicht nach gesetzlichen Vorschriften erlaubt ist, ist für die oben aufgeführten Weiterverwendungen des Materials die Einwilligung des jeweiligen Rechteinhabers einzuholen.

Weitere Details zur Lizenz entnehmen Sie bitte der Lizenzinformation auf http://creativecommons.org/ licenses/by/4.0/deed.de.

\section{Literatur}

Adler, F. (2016). Transformation zur Postwachstumsgesellschaft - ja, aber wie? Kolleg Postwachstumsgesellschaft, Working Paper 3/2016. Jena: Universität Jena.

Adloff, F., \& Neckel, S. (2019). Futures of sustainability as modernization, transformation and control: A conceptual framework. Sustainability Science, 14, 1015-1025.

Baumann, Z. (2003). Flüchtige Moderne. Frankfurt a. M.: Suhrkamp.

Berg, C. (2020). Ist Nachhaltigkeit utopisch? Wie wir Barrieren überwinden und zukunftsfähig handeln. München: oekom.

Blättel-Mink, B. (2016). Konsum und Nachhaltigkeit. In C. Besio \& G. Romano (Hrsg.), Zum gesellschaftlichen Umgang mit dem Klimawandel (S. 241-268). Baden-Baden: Nomos.

Blühdorn, I. (2000). Post-ecological politics. Social theory and the abdication of the ecologist paradigm. London: Routledge.

Blühdorn, I. (2011). The politics of unsustainability: COP15, post-ecologism, and the ecological paradox. Organization \& Environment, 24, 34-53.

Blühdorn, I. (2013). Simulative Demokratie. Neue Politik nach der postdemokratischen Wende. Berlin: Suhrkamp.

Blühdorn, I. (2017). Post-capitalism, post-growth, post-consumerism? Eco-political hopes beyond sustainability. Global Discourse, 7, 42-61.

Blühdorn, I. (2018). Nicht-Nachhaltigkeit auf der Suche nach einer politischen Form. Konturen der demokratischen Postwachstumsgesellschaft. Berliner Journal für Soziologie, 28, 151-180.

Blühdorn, I. (2019). „Wir werden der Frage nach Begrenzung von Freiheiten nicht entkommen. “ Interview von Markus C. Schulte von Drach mit Ingolfur Blühdorn. Süddeutsche Zeitung vom 19. Oktober 2019. https://www.sz.de/1.4625111. Zugegriffen: Mai 2021.

Blühdorn, I. (2020a). Haben wir es gewollt? Vorüberlegungen. In I. Blühdorn, F. Butzlaff, M. Deflorian, D. Hausknost \& M. Mock, Nachhaltige Nicht-Nachhaltigkeit. Warum die ökologische Transformation der Gesellschaft nicht stattfindet (S. 13-27). Bielefeld: transcript. 
Blühdorn, I. (2020b). Kein gutes Leben für Alle. Annäherung an einen Paradigmenwechsel. In I. Blühdorn, F. Butzlaff, M. Deflorian, D. Hausknost \& M. Mock, Nachhaltige Nicht-Nachhaltigkeit. Warum die ökologische Transformation der Gesellschaft nicht stattfindet (S. 29-64). Bielefeld: transcript.

Blühdorn, I. (2020c). Die Gesellschaft der Nicht-Nachhaltigkeit. Skizze einer umweltsoziologischen Gegenwartsdiagnose. In I. Blühdorn, F. Butzlaff, M. Deflorian, D. Hausknost \& M. Mock, Nachhaltige Nicht-Nachhaltigkeit. Warum die ökologische Transformation der Gesellschaft nicht stattfindet (S. 65-142). Bielefeld: transcript.

Blühdorn, I. (2020d). Demokratie der Nicht-Nachhaltigkeit. Begehung eines umweltsoziologischen Minenfelds. In I. Blühdorn, F. Butzlaff, M. Deflorian, D. Hausknost \& M. Mock, Nachhaltige Nicht-Nachhaltigkeit. Warum die ökologische Transformation der Gesellschaft nicht stattfindet (S. 287-327). Bielefeld: transcript.

Blühdorn, I., Butzlaff, F., Deflorian, M., Hausknost, D., \& Mock, M. (2020). Nachhaltige Nicht-Nachhaltigkeit. Warum die ökologische Transformation der Gesellschaft nicht stattfindet. Bielefeld: transcript.

Boltanski, L., \& Chiapello, È. (2003). Der neue Geist des Kapitalismus. Konstanz: UVK.

Bourdieu, P. (1982). Die feinen Unterschiede. Kritik der gesellschaftlichen Urteilskraft. Frankfurt a. M.: Suhrkamp.

Brand, K.-W. (2008). Konsum im Kontext. Der „,verantwortliche Konsument“ - ein Motor nachhaltigen Konsums? In H. Lange (Hrsg.), Nachhaltigkeit als radikaler Wandel. Die Quadratur des Kreises? (S. 71-93). Wiesbaden: VS Verlag für Sozialwissenschaften.

Brand, K.-W. (2010). Die Neuerfindung des Bürgers. Soziale Bewegungen und bürgerschaftliches Engagement in der Bundesrepublik. In T. Olk, A. Klein \& B. Hartnuß (Hrsg.), Engagementpolitik. Die Entwicklung der Zivilgesellschaft als politische Aufgabe (S. 123-152). Wiesbaden: VS Verlag für Sozialwissenschaften.

Brand, K.-W. (2011). Umweltsoziologie und der praxistheoretische Zugang. In M. Groß (Hrsg.), Handbuch Umweltsoziologie (S. 173-198). Wiesbaden: VS Verlag für Sozialwissenschaften.

Brand, K.-W. (Hrsg.). (2017). Die sozial-ökologische Transformation der Welt. Ein Handbuch. Frankfurt a. M.: Campus.

Brand, K.-W. (2018). Disruptive Transformationen. Gesellschaftliche Umbrüche und sozial-ökologische Transformationsdynamiken kapitalistischer Industriegesellschaften - ein zyklisch-struktureller Ansatz. Berliner Journal für Soziologie, 28, 479-509.

Brand, K.-W. (2020). Nachhaltigkeitsperspektiven in der (Post-)Corona Welt. Globale Umbrüche und die Herausbildung neuer Resilienzregime. SuN - Soziologie und Nachhaltigkeit, Sonderband II: Die sozial-ökologische Transformation in der Corona-Krise, 8-20. https://doi.org/10.17879/sun-2020-2937.

Brand, K.-W. (2021). „Große Transformation“ oder „Nachhaltige Nicht-Nachhaltigkeit“? Wider die Beliebigkeit sozialwissenschaftlicher Nachhaltigkeits- und Transformationstheorien. Leviathan, 49, 189-214.

Brand, K.-W., Büsser, D., \& Rucht, D. (1986). Aufbruch in eine andere Gesellschaft. Neue soziale Bewegungen in der Bundesrepublik. Frankfurt a. M.: Campus.

Bröckling, U. (2007). Das unternehmerische Selbst. Soziologie einer Subjektivierungsform. Frankfurt a. M.: Suhrkamp.

CBD (Secretariat of the Convention on Biological Diversity) (2020). Global biodiversity outlook 5. Montreal: CBD.

Conrad, S., \& Randeria, S. (Hrsg.). (2002). Jenseits des Eurozentrismus. Postkoloniale Perspektiven in den Geschichts- und Kulturwissenschaften. Frankfurt a. M.: Campus.

Crouch, C. (2008). Postdemokratie. Frankfurt a. M.: Suhrkamp.

Dörre, K. (2020). Die Corona-Pandemie - eine Katastrophe mit Sprengkraft. Berliner Journal für Soziologie, 30, 165-190.

Dörre, K., Rosa, H., Becker, K., Bose, S., \& Seyd, B. (Hrsg.). (2019). Große Transformation? Zur Zukunft moderner Gesellschaften. Sonderband des Berliner Journals für Soziologie. Wiesbaden: Springer VS.

Enquete-Kommission „Zukunft des bürgerschaftlichen Engagements“ der Deutschen Bundestages (2002). Bericht. Bürgerschaftliches Engagement: Auf dem Weg in eine zukunftsfähige Bürgergesellschaft. Opladen: Leske + Budrich.

Geiselberger, H. (Hrsg.). (2016). Die große Regression. Eine internationale Debatte über die geistige Situation der Zeit. Berlin: Suhrkamp.

Giddens, A. (1988). Die Konstitution der Gesellschaft. Grundzüge einer Theorie der Strukturierung. Frankfurt a. M.: Campus.

Görg, C., Brand, U., Haberl, H., Hummel, D., Jahn, T., \& Liehr, S. (2017). Challenges for social-ecological transformations: Contributions from social and political ecology. Sustainability, 9, https://doi.org/10. 3390/su9071045. 
Grin, J., Rotman, J., \& Schot, J. (2010). Transitions to sustainable development. New directions in the study of long term transformative change. New York: Routledge.

Grunwald, A. (2012). Ende einer Illusion. Warum ökologisch korrekter Konsum die Umwelt nicht retten kann. München: oekom.

Hausknost, D. (2020). Die gläserne Decke der Transformation. Strukturelle Blockaden im demokratischen Staat. In I. Blühdorn, F. Butzlaff, M. Deflorian, D. Hausknost \& M. Mock, Nachhaltige Nicht-Nachhaltigkeit. Warum die ökologische Transformation der Gesellschaft nicht stattfindet (S. 143-171). Bielefeld: transcript.

Hirschman, A. O. (1982). Shifting involvements. Private interests and public action. Princeton: Princeton University Press.

Katz, C., \& von Winterfeld, U. (2006). Im Schatten der Aufklärung. Zur Kontinuität der Natur- und Geschlechterkonstruktionen von Bacon bis Brundtland. In W. Ernst \& U. Bohle (Hrsg.), Naturbilder und Lebensgrundlagen. Konstruktionen von Geschlecht (S. 194-232). Hamburg: LIT.

Kemp, R., Loorbach, D., \& Rotmans, J. (2007). Transition management as a model for managing processes of co-evolution towards sustainable development. The International Journal of Sustainable Development and World Ecology, 14, 78-91.

Köhler, G. (2015). Die Millenniums-Entwicklungsziele - ein kritischer Rückblick und optimistischer Ausblick. Vereinte Nationen, (6), 243-248.

Kollmorgen, R., Merkel, W., \& Wagner, H.-J. (Hrsg.). (2015). Handbuch Transformationsforschung. Wiesbaden: Springer VS.

Konzeptwerk Neue Ökonomie \& DFG-Kolleg Postwachstumsgesellschaften (Hrsg.). (2017). Degrowth in Bewegung(en). 32 alternative Wege zur sozial-ökologischen Transformation. München: oekom.

Kriesi, H., Koopman, R., Duyvendak, J., \& Guigni, M. (1995). The new social movements in Western Europe. A comparative analysis. Minneapolis: University of Minnesota Press.

Kropp, C. (2019). Nachhaltige Innovationen. In B. Blättel-Mink, I. Schulz-Schaeffer \& A. Windeler (Hrsg.), Handbuch Innovationsforschung. Wiesbaden: Springer VS. https://doi.org/10.1007/978-3658-17671-6_50-1.

Kungl, G. (2021). Ein grüner Geist des Kapitalismus? Konturen einer neuen Wirtschaftsgesinnung. SOI Discussion Paper 2021-01. Stuttgart: Universität Stuttgart.

Latour, B. (1998). Wir sind nie modern gewesen. Versuch einer symmetrischen Anthropologie. Frankfurt a. M.: Fischer.

Lessenich, S. (2008). Die Neuerfindung des Sozialen. Der Sozialstaat im flexiblen Kapitalismus. Bielefeld: transcript.

Lessenich, S. (2020). Soziologie - Corona - Kritik. Berliner Journal für Soziologie, 30, 215-230.

Merchant, C. (1987). Der Tod der Natur. Ökologie, Frauen und neuzeitliche Naturwissenschaft. München: C. H. Beck.

Mock, M. (2020). Verantwortliches Individuum? Die (Un-)Haltbarkeit der Erzählung von der Konsument*innenverantwortung. In I. Blühdorn, F. Butzlaff, M. Deflorian, D. Hausknost \& M. Mock, Nachhaltige Nicht-Nachhaltigkeit. Warum die ökologische Transformation der Gesellschaft nicht stattfin$\operatorname{det}$ (S. 227-253). Bielefeld: transcript.

Neckel, S. (2018). Ökologische Distinktion. In S. Neckel, N. Besedovsky, M. Boddenberg, M. Hasenfratz, S. M. Pritz \& T. Wiegand, Die Gesellschaft der Nachhaltigkeit. Umrisse eines Forschungsprogramms (S. 59-76). Bielefeld: transcript.

Neckel, S. (2020). Der Streit um die Lebensführung. Nachhaltigkeit als sozialer Konflikt. Mittelweg 36, 29(6), 82-100.

Neckel, S., Besedovsky, N., Boddenberg, M., Hasenfratz, M., Pritz, S. M., \& Wiegand, T. (2018). Die Gesellschaft der Nachhaltigkeit. Umrisse eines Forschungsprogramms. Bielefeld: transcript.

Oxfam (2019). Public good or private wealth? Oxford: Oxfam.

Oxfam (2021). Das Ungleichheitsvirus. Wie die Corona-Pandemie soziale Ungleichheit verschärft und warum wir unsere Wirtschaft gerechter gestalten müssen. Berlin: Oxfam Deutschland.

Rancière, J. (1996). Demokratie und Postdemokratie. In A. Badiou \& J. Rancière, Politik der Wahrheit (S. 119-156). Wien: Turia + Kant.

Reckwitz, A. (2003). Grundelemente einer Theorie sozialer Praktiken: Eine sozialtheoretische Perspektive. Zeitschrift für Soziologie, 32, 282-301.

Reckwitz, A. (2006). Das hybride Subjekt. Eine Theorie der Subjektkulturen von der bürgerlichen Moderne zur Postmoderne. Weilerswist: Velbrück.

Reckwitz, A. (2017). Die Gesellschaft der Singularitäten. Berlin: Suhrkamp.

Reckwitz, A. (2019). Das Ende der Illusionen. Politik, Ökonomie und Kultur in der Spätmoderne. Berlin: Suhrkamp. 
Reißig, R. (2009). Gesellschafts-Transformation im 21. Jahrhundert. Ein neues Konzept sozialen Wandels. Wiesbaden: VS Verlag für Sozialwissenschaften.

Rosa, H. (2020). Pfadabhängigkeit, Bifurkationspunkte und die Rolle der Soziologie. Ein soziologischer Deutungsversuch der Corona-Krise. Berliner Journal für Soziologie, 30, 191-213.

Rückert-John, J., \& Schäfer, M. (Hrsg.). (2017). Governance für eine Gesellschaftstransformation. Herausforderungen des Wandels in Richtung nachhaltige Entwicklung. Wiesbaden: Springer VS.

Schatzki, T. (1996). Social practices: A Wittgensteinian approach to human activity and the social. Cambridge (US): Cambridge University Press.

Schmalz, S. (2018). Machtverschiebungen im Weltsystem. Der Aufstieg Chinas und die große Krise. Frankfurt a. M.: Campus.

Schneidewind, U. (2018). Die Große Transformation. Eine Einführung in die Kunst gesellschaftlichen Wandels. Frankfurt a. M.: Fischer.

Shove, E. (2003). Comfort, cleanliness and convenience. The social organisation of normality. London: Berg.

Shove, E., Watson, M., Hand, M., \& Ingram, J. (2007). The design of everyday life. Oxford: Berg.

SONA - Netzwerk Soziologie der Nachhaltigkeit (Hrsg.). (2021). Soziologie der Nachhaltigkeit. Bielefeld: transcript.

Steffen, W., Richardson, K., Rockström, J., et al. (2015), Planetary boundaries: Guiding human development on a changing planet. Science, 347(6223), https://doi.org/10.1126/science.1259855.

UN (United Nations) (2015). The millennium development goals report. New York: United Nations.

WBGU (Wissenschaftlicher Beirat der Bundesregierung Globale Umweltveränderungen) (2011). Welt im Wandel. Gesellschaftsvertrag für eine Große Transformation. Berlin: WBGU.

Welsch, W. (1990). Unsere postmoderne Moderne. Berlin: Akademie Verlag.

Zilleßen, H., Dienel, P., \& Strubelt, W. (Hrsg.). (1993). Die Modernisierung der Demokratie. Internationale Ansätze. Wiesbaden: Westdeutscher Verlag.

Karl-Werner Brand geb. 1944. Prof. i. R. an der Technischen Universität München. Langjähriger Leiter der Münchner Projektgruppe für Sozialforschung (MPS). Derzeit freiberuflich tätig als Lehrbeauftragter für Umweltsoziologie sowie im Rahmen der Nachhaltigkeitsforschung (Sustainability Research Consulting). Forschungsschwerpunkte: Umweltsoziologie, sozialwissenschaftliche Nachhaltigkeits- und Transformationsforschung, Praxistheorie, politische und historische Soziologie, soziale Bewegungsforschung. Ausgewählte aktuelle Veröffentlichungen: (Hrsg.) Die sozial-ökologische Transformation der Welt. Ein Handbuch, 2017; Disruptive Transformationen. Gesellschaftliche Umbrüche und sozial-ökologische Transformationsdynamiken kapitalistischer Industriegesellschaften - ein zyklisch-struktureller Erklärungsansatz, in: Berliner Journal für Soziologie, 2018; (mit K. Block et al.) Soziologie der Nachhaltigkeit. Zwischen Transformation und Reflexion, in: Soziologie und Nachhaltigkeit, 2018; „Große Transformation“ oder „Nachhaltige Nicht-Nachhaltigkeit"? Wider die Beliebigkeit sozialwissenschaftlicher Nachhaltigkeits- und Transformationstheorien, in: Leviathan, 2021. 